\title{
Investment Timing and Incentive Costs*
}

\author{
Sebastian Gryglewicz ${ }^{\dagger}$ and Barney Hartman-Glaser ${ }^{\ddagger}$
}

March 18, 2019

\begin{abstract}
We analyze how the costs of smoothly adjusting capital, such as incentive costs, affect investment timing. In our model, the owner of a firm holds a real option to increase a lumpy form of capital and can also smoothly adjust an incremental form of capital. Increasing the cost of incremental capital can delay or accelerate investment in lumpy capital. Incentive costs due to moral hazard are a natural source of costs for the accumulation of incremental capital. When moral hazard is severe, delaying investment in lumpy capital is costly, and it is optimal to overinvest relative to the first-best case.
\end{abstract}

JEL classification: G31, D92, D86.

Keywords: Moral Hazard, Dynamic Contracting, Real Options.

${ }^{*}$ We sincerely thank the editor Itay Goldstein and the two anonymous referees. We are also grateful for the helpful feedback from Hengjie Ai, Ulf Axelson, Jonathan Berk, Antonio Bernardo, Bruno Biais, Andrea Buffa, Bruce Carlin, Peter DeMarzo, Andrea Eisfeldt, Mark Garmaise, Simon Gervais, Steven Grenadier, Valentin Haddad, Zhiguo He, David Hirshleifer, Dmitry Livdan, Semyon Malamud, William Mann, Erwan Morellec, Kevin Murphy, Boris Nikolov, Paul Pfleiderer, Norman Schürhoff, Avandihar (Subra) Subrahmanyam, Alexei Tchistyi, Vish Vishwanathan, Nancy Wallace, Francesca Zucchi, and Gijsbert Zwart. We also wish to thank the seminar and conference participants at UC Berkeley, Duke, CPB Netherlands, Lausanne-EPFL, the Revelstoke Finance Summit, VU Amsterdam, the SITE Summer Workshop, Erasmus, the Minnesota Junior Finance Conference, UCLA, Amsterdam, Aarhus, the Adam Smith Corporate Finance Conference, the USC-UCLA-UCI Finance Day, the SFS Finance Cavalcade, Stanford GSB, the EEA Meetings, the EFA Meetings, Cal Poly San Luis Obispo, and the WFA Meetings for their useful comments and suggestions. All errors are our own. An earlier version of this paper was titled Dynamic Agency and Real Options.

${ }^{\dagger}$ Erasmus School of Economics, Erasmus University Rotterdam, Rotterdam, The Netherlands, Phone: +31-104081435, E-mail: gryglewicz@ese.eur.nl

${ }^{\ddagger}$ Anderson School of Management, University of California at Los Angeles, Los Angeles, CA, Phone: 310-825-4083, E-mail: bhglaser@anderson.ucla.edu 


\section{Introduction}

Financial economists often view patterns of inefficient investment through the prism of agency conflicts. For example, in his seminal paper, Jensen (1986) posits that managers' private benefits of investment, i.e., empire-building preferences, cause firms to overinvest relative to outside shareholders' preferences. In contrast, a substantial body of literature links underinvestment to managers' private costs of firm operations. For example, DeMarzo and Fishman (2007) show that managers' ability to divert cash flow decreases the net return of adding new capital and thus lowers investment. As such, a dichotomy has emerged in the literature: overinvestment is a result of managers' private benefits of investment, whereas underinvestment is a result of their private costs of firm operations. ${ }^{1}$ The main contribution of this paper is to challenge this dichotomy and show that a manager's ability to shirk or divert cash flow, a form of private costs of firm operations, can accelerate investment when investment technology is lumpy.

We specify a dynamic model of investment in which a firm optimally accumulates two inputs of production. The firm can smoothly adjust the first input, such as productivity or organization capital, at a total cost that derives from both direct expenditure and from incentives for the firm's manager. Specifically, the manager needs incentives to prevent her from diverting funds allocated to cover the direct cost. The firm also has an option to invest in a discrete amount of capital, such as a new factory or an acquisition. The two inputs are complements in the production function: an increase in the quantity of one in the firm increases the marginal productivity of the other. It is then optimal to delay exercising the option until the output has reached an endogenous investment threshold. For convenience, we call the first and second inputs incremental and lumpy capital, respectively.

An increase in the severity of the moral hazard problem, and thus the cost of incremental capital investment, has a non-monotonic effect on the optimal investment threshold for the real option to increase lumpy capital. When the cost is low, increasing it leads to a delay in the optimal time to exercise the lumpy capital expansion option. The intuition for this case is straightforward. Increasing the cost of incremental capital reduces both its optimal growth rate and the value of investing in lumpy capital because the two forms of capital are complements in the production

\footnotetext{
${ }^{1}$ Stein (2003) provides a thorough review of the literature on empire building. Tirole (2010) provides a treatment of the effects of the private costs of firm operations on investment.
} 
function of the firm. In general terms, the agency conflict makes lumpy capital less productive, thus curtailing investment, as in much of the literature on operational conflicts and investment, such as DeMarzo, Fishman, He, and Wang (2012). This intuition only applies to our setting when the cost of investment in incremental capital is low.

When the cost of investment in incremental capital is high, increasing it can accelerate investment in lumpy capital. Before the exercise of the option to invest in lumpy capital, the optimal growth rate of incremental capital takes two benefits into account. First, additional incremental capital increases contemporaneous cash flow. Second, it increases the value of the option to add lumpy capital. After investors exercise the option, only the first benefit remains. Thus, the optimal investment rate in incremental capital can decrease after lumpy capital expansion. When the cost of investment in incremental capital is large, i.e., when the moral hazard problem is severe, this decrease is also large. For example, if the cost is large enough the optimal investment rate in incremental capital is positive before the exercise of the growth option and zero afterward. In this case, an increase in the cost of investment in incremental capital decreases net cash flow before the option exercise and leaves net cash flow unchanged afterward. This in turn increases the marginal cost of delaying the exercise of the real option and lowers the investment threshold.

By treating moral hazard as an additional cost of investing in incremental capital, we can contribute to the debate on the causes of over- vs. underinvestment. First, our reasoning above is not specific to moral hazard costs. We can decompose the total cost of investing in incremental capital into two components: direct costs that are given by the resource expenditure required to acquire new incremental capital and incentive costs that are given by the additional cost of acquiring incremental capital through an agent. Increasing the direct costs can have the same effects discussed above even without moral hazard - in other words, under the first-best case. However, when the incentive costs are high relative to the direct costs, the investment threshold is lower under moral hazard than under the first-best case. This behavior is a form of overinvestment in that the firm exercises the growth option earlier than it otherwise would if not for the incentive costs. In this way, a manager's private costs of firm operations - i.e., consuming the resources required to grow incremental capital on her own account - can cause overinvestment.

Beyond showing that a manager's private costs of firm operations can cause overinvestment, we also argue that incentive costs are an empirically relevant source of costs for the smooth accumu- 
lation of capital. Whereas capital investments made in lumpy fashion, such as building an entire plant, are likely to be readily observable and subject to contracts, investment in small increments, such as adding or maintaining machinery at existing plants, is more likely to be subject to managerial discretion and hence to moral hazard. Managers can divert resources meant for incremental investment for their consumption, as such resources are difficult to measure and quantify in real time. As a result, an empirical examination of the interaction between incremental investment and the exercise of large real options requires an understanding of the role moral hazard plays in the accumulation of capital. This stance is broadly consistent with empirical evidence on acquisitions, such as in Datta, Iskandar-Datta, and Raman (2001) and Harford and Li (2007), showing that acquisition decisions are related to managerial incentives, both before and after acquisitions.

An important difference between incentive costs and the direct cost of incremental capital lies in how uncertainty affects the optimal exercise policy for the lumpy capital expansion option. In our model, uncertainty is represented by the volatility of capital depreciation shocks. The effect of a change in this volatility acts through two channels. First, as in a classic real option exercise problem, increasing volatility increases the value of the option to wait to invest and thus lowers the investment threshold. Second, the moral hazard problem leads to an endogenous link between volatility and incentive costs. When volatility is higher, providing the manager with incentives for investment in incremental capital requires her to take on more risk, which in turn increases incentive costs. When the moral hazard problem is already severe, the second effect dominates the first, and an increase in volatility decreases the optimal investment threshold. This result cannot be obtained without moral hazard, as in that case volatility does not affect the cost of incremental capital investment.

Our results are consistent with several findings in the empirical literature on large investments. In particular, our model offers a framework for interpreting observed patterns of managerial compensation and firm performance around acquisitions. For example, Harford and Li (2007) study the dynamics of executive incentives around acquisition events and find that incentives change substantially around merger events with a decrease in pay-performance sensitivity. We can interpret the lumpy capital investment option in our model as an option to acquire another firm, in which case our model implies that the strength of incentives should occur post-merger to decrease the optimal rate of investment in incremental growth. Harford and Li (2007) also find that firm performance 
suffers post-acquisition, which is consistent with a decrease in incremental growth after a large investment. Titman, Wei, and Xie (2004) find that firms tend to have poor long-term performance after undertaking large capital expenditures. If large capital expenditures are indicative of the exercise of growth opportunities, then these results are again consistent with our finding of a drop in incremental growth following large investments.

Our results also shed light on the choice between innovation through internal investment and innovation through acquisitions. Importantly, these two forms of innovation are often complements: more productive firms can more efficiently exploit technology that they acquire externally, as documented by Cassiman and Veugelers (2006). The complementarity of internal and external innovation would seem to indicate that firms should actively invest in both forms of innovation. However, mature firms tend to innovate through acquisitions while young firms tend to innovate via incremental internal investment (Huergo and Jaumandreu (2004) and Zhao (2009)). This pattern is consistent with our model if more mature firms are subject to more severe agency conflicts. Relatedly, Balsmeier, Fleming, and Manso (2017) show that agency conflicts negatively affect innovation. In this context, the model implies that industries characterized by a large number of mature firms will feature frequent acquisitions, as is currently the case in the technology industry. ${ }^{2}$

This paper contributes to the literature on corporate investment under uncertainty. Our model of real options is based on the seminal work of McDonald and Siegel (1986), Brennan and Schwartz (1985), and Abel and Eberly (1996). In the classic real options literature, growth, either in cash flows or productivity, is taken as exogenous. In our model, the firm's owners and managers must expend resources to grow the prospects of the firm.

This paper also contributes to the growing literature on the intersection of dynamic agency conflicts and investment under uncertainty. On the dynamic contracting side, Holmstrom and Milgrom (1987) and Spear and Srivastava (1987) introduce the notion that agents may be provided with incentives over many periods. More recently, there has been renewed interest in dynamic contracting. Biais, Mariotti, Plantin, and Rochet (2007) analyze a rich discrete-time model of a dynamic agency conflict and its continuous-time limit. We adopt the continuous-time framework of He (2011). ${ }^{3}$ On the investment side, DeMarzo and Fishman (2007), Biais, Mariotti, Rochet,

\footnotetext{
${ }^{2}$ See, for example, "American tech giants are making life tough for startups" in The Economist, June 2, 2018.

${ }^{3}$ For more examples of continuous-time dynamic contracting, see Sannikov (2008), DeMarzo and Sannikov (2006), Piskorski and Tchistyi (2010, 2011), and He (2009).
} 
and Villeneuve (2010), and DeMarzo et al. (2012) consider dynamic moral hazard with investment. One important distinction between our paper and both Biais et al. (2010) and DeMarzo et al. (2012) is that in their models, the first-best effort is optimal even under moral hazard. Malenko (2018) also studies a dynamic capital-budgeting problem, but in contrast to much of the literature, directly considers managerial empire-building preferences. Grenadier, Malenko, and Malenko (2016) study how strategic communication between a firm's managers and its owners can distort the optimal timing of real options. Grenadier and Malenko (2011) show that managers may distort the real option exercise to signal private information to investors. In subsequent work, Gryglewicz, Hartman-Glaser, and Zheng (2018) use a similar model to the one used in this paper to show that pay-performance sensitivity can decrease with the increasing intensity of growth options, and they provide empirical evidence consistent with this prediction.

Grenadier and Wang (2005) study how agency conflicts can affect the exercise of real options. In their model, the owner of a real option delegates the investment timing decision to a manager who can exert effort to increase the payoff of the option. The manager also privately observes this payoff. In this setting, the manager has an incentive to report that the option has low value and to consume the difference between the true value of the project and her report. Under the optimal contract, the owner sets the investment threshold for low-value projects higher than in the first-best case to dissuade the manager from under-reporting project quality. In our model, there is no conflict of interest between the owner of the firm and the manager over investment timing. We also assume that the cost of the moral hazard problem depends directly on when the option is exercised. Importantly, this second feature means that incentivizing managerial effort affects the real option exercise without assuming any additional conflicts of interest.

Philippon and Sannikov (2007) consider real options in a dynamic moral hazard setting similar to ours. In their model, cash flows follow an i.i.d. process, and hence there is no real option problem under the first-best case. That is, in the first-best case, the firm always immediately invests, as the investment is assumed to have a positive net present value. Introducing the agency problem in their setting induces a valuable option to delay investment until the agent has sufficiently high continuation utility and the firm is very unlikely to be liquidated. Consequently, moral hazard can only delay investment in their setting. In contrast, we model cash flows that grow in expectation, and as a consequence, optimal managerial effort depends on the level of cash flows where investment 
and effort may serve as substitutes. This difference means that in our model, unlike in that of Philippon and Sannikov, moral hazard can either raise or lower the investment threshold.

\section{A Model of Incremental and Lumpy Investment}

In this section, we present a model of investment in which a firm produces cash flow using two forms of capital. The first form of capital can be adjusted via a neoclassical investment technology. The second form of capital can be increased by a discrete amount in the manner of a standard real option to invest. Our analysis characterizes how changes to the cost of investing in the first form of capital affect the optimal timing of investing in the second.

\section{$2.1 \quad$ Setup}

Time is continuous, infinite, and indexed by $t$. The risk-free rate is $r$. A risk-neutral investor owns a firm that produces cash flow $Y_{t} d t$ using lumpy capital $K_{t}$, which can only be added in a discrete amount, and incremental capital $X_{t}$, which can be smoothly adjusted according to the following production function: ${ }^{4}$

$$
Y_{t}=X_{t} K_{t}
$$

The firm starts with $K_{0}=k_{\mathrm{s}}>0$ units of lumpy capital. The investor has a one-time option to increase lumpy capital to $k_{\mathrm{b}}>k_{\mathrm{s}}$ at cost $P=p\left(k_{\mathrm{b}}-k_{\mathrm{s}}\right)$, where $p$ is the per-unit price. ${ }^{5}$ We let $\tau$ denote the stopping time at which the investor exercises this option.

Incremental capital $X_{t}$ accumulates according to the following dynamics:

$$
d X_{t}=I_{t} d t+\sigma X_{t} d Z_{t}
$$

where $I_{t} \geq 0$ is the investment in incremental capital and $Z_{t}$ is the standard Brownian motion driving incremental capital depreciation shocks. Investment in $X$ incurs a cost $F(I, x, k)=G(I, x) k$ per unit of time and can include both the direct price of acquiring the capital and the adjustment

\footnotetext{
${ }^{4}$ In Appendix C.3, we consider a version of the model with a single form of capital that can be adjusted using both investment technologies described above. The results are similar. However, distinguishing between the two forms of capital facilitates the economic interpretation of the primitives of the model.

${ }^{5}$ In Appendix C.4, we consider a version of the model in which a firm holds an option to abandon instead of an option to invest.
} 
cost. This specification for the cost of investment captures the notion that it is more costly to increase incremental capital when the firm uses more lumpy capital. It is convenient to represent investment in incremental capital as a per-unit rate, $i_{t}=I_{t} / X_{t}$, and rewrite the dynamics of $X_{t}$ as follows:

$$
d X_{t}=i_{t} X_{t} d t+\sigma X_{t} d Z_{t}
$$

We assume that $G(I, X)$ is homogenous of degree one in $I$ and $X$, allowing the total investment cost to be written as follows:

$$
F(I, x, k)=\theta g(i) x k
$$

for some function $g$ and some constant $\theta>0$. We assume that this rate must be positive and cannot exceed some upper limit such that $i_{t} \in\left[0, i_{\max }\right]$, where $i_{\max }<r$. Finally, we assume that $F$ is such that $g(i)$ is continuous and $g(i) \geq 0, g^{\prime}(i)>0$. To simplify the analysis, we also assume that either $g^{\prime \prime}(i)=0$ or $g^{\prime \prime}(i)>0$ for all $i$. Note that this production technology implies that $X$ and $K$ are complements in the production function in that increasing the quantity of one improves the marginal productivity of the other. For a more formal discussion of the complementarity between $X$ and $K$, see Appendix C.1.

Given the technology described above, the investor's problem is to choose an investment policy $i$ in incremental capital and a time $\tau$ to exercise the real option to expand lumpy capital to maximize the present value of the firm's cash flow net of investment costs:

$$
\max _{i, \tau} E\left[\int_{0}^{\infty} e^{-r t}\left(X_{t} K_{t}-\theta g\left(i_{t}\right) X_{t} K_{t}\right) d t-e^{-r \tau} P\right]
$$

\subsection{Model Solution}

We take the standard dynamic programming approach to solve the investor's problem in Equation (4). First, we characterize the optimal investment rate for incremental capital. Over any region of $x$ such that the investor does not exercise the real option, an application of Ito's formula together with the dynamic programming principle implies the following Hamilton-Jacobi-Bellman (HJB) equation for the value of the firm $V(x, k)$ :

$$
r V=\max _{i \in\left[0, i_{\max }\right]}\left\{x k(1-\theta g(i))+i x \frac{\partial V}{\partial x}+\frac{1}{2} \sigma^{2} x^{2} \frac{\partial^{2} V}{\partial x^{2}}\right\} .
$$


The right-hand side of Equation (5) is the sum of the cash flow generated by the firm net of the cost of investing in incremental capital and the expected capital gains in firm value from this investment. Let $\hat{i}(x, k)$ be the solution to a first-order condition for Equation $(5)^{6}$ :

$$
\theta g^{\prime}(\hat{i}) k=\frac{\partial V}{\partial x}
$$

Then, the optimal investment rate, denoted by $i^{*}(x, k)$, is given by the following:

$$
i^{*}(x, k)= \begin{cases}0 & \text { if } \theta g^{\prime}(0) k \geq \frac{\partial V}{\partial x}, \\ i_{\max } & \text { if } \theta g^{\prime}\left(i_{\max }\right) k \leq \frac{\partial V}{\partial x}, \text { and } \\ \hat{i}(x, k) & \text { otherwise. }\end{cases}
$$

Next, we characterize the optimal time at which to exercise the real option to expand lumpy capital. As is common in real options to invest, the optimal exercise policy takes the form of a threshold rule, $\tau=\inf \left\{t: X_{t} \geq \bar{x}\right\}$. The location of the exercise threshold is identified using the following value-matching and smooth-pasting conditions:

$$
\begin{aligned}
V\left(\bar{x}, k_{\mathrm{s}}\right) & =V\left(\bar{x}, k_{\mathrm{b}}\right)-P \\
\frac{\partial}{\partial x} V\left(\bar{x}, k_{\mathrm{s}}\right) & =\frac{\partial}{\partial x} V\left(\bar{x}, k_{\mathrm{b}}\right) .
\end{aligned}
$$

Finally, the value of the firm must be zero when incremental capital falls to zero:

$$
V(0, k)=0
$$

We summarize our results about the firm value function and optimal policies in the proposition below.

Proposition 1. The value of the firm $V(x, k)$, the optimal investment rate $i^{*}$ in incremental capital, and the optimal exercise time $\tau$ of the option to expand lumpy capital are provided by the solution to Equation (5) with the boundary conditions in Equations (8) to (10).

\footnotetext{
${ }^{6}$ If $g^{\prime \prime}(i)>0$, then Equation (6) has a unique solution. If $g^{\prime \prime}(i)=0$, then $i^{*} \in\left\{0, i_{\max }\right\}$.
} 


$$
V\left(x, k_{\mathrm{b}}\right)=\frac{1-\theta g\left(i^{*}\left(x, k_{\mathrm{b}}\right)\right)}{r-i^{*}\left(x, k_{\mathrm{b}}\right)} x k_{\mathrm{b}}
$$

where $i^{*}\left(x, k_{\mathrm{b}}\right)$, if it is interior, solves the following

$$
\theta g^{\prime}\left(i^{*}\right)=\frac{1-\theta g\left(i^{*}\right)}{r-i^{*}}
$$

Whether analytical solutions for the pre-exercise firm value $V\left(x, k_{\mathrm{s}}\right)$ and optimal exercise threshold $\bar{x}$ exist depends on the structure of the cost of investing in incremental capital. We analyze various cases in the next sections. In each case, we study the effect of an increase in the cost of investment in incremental capital on the optimal exercise threshold for the real option.

\section{The Cost of Incremental Capital and the Real Option Exercise}

In this section, we consider the effect of optimal investment in incremental capital on the optimal real option exercise policy in the context of increasingly rich specifications of the model that we describe in Section 2. To begin our analysis, we study a pure real option problem in which the investment in incremental capital only plays a role after the real option to expand lumpy capital has been exercised. In the following subsections, we allow investment in incremental capital to enter the investor's problem both before and after the exercise of the real option. We show that the presence of this additional decision means that increasing the cost of incremental capital can lead to a lower optimal exercise threshold for the option to expand lumpy capital.

\subsection{Real Option to Initiate a Project}

In this section, we consider the problem in which the firm starts with zero lumpy capital. When $k_{\mathrm{s}}=0$, the cost of investment in incremental capital is zero before exercising the real option, and it is therefore always optimal to set $i^{*}\left(x, k_{s}\right)=i_{\max }$. Here, our model resembles a classic real option problem of when to initiate a project in the spirit of McDonald and Siegel (1986). The firm starts life producing zero cash flows and incurring no costs. Incremental capital grows at a fixed rate $i_{\max }$ in expectation. Once the firm has accumulated enough incremental capital, there is a time at which it is optimal to begin the project by investing in lumpy capital. The main difference between 
our model and the classic real option problem is that in our model, unlike the standard model, initiating the project substantially changes the cost of future growth in incremental capital. As a result, the cost of investing in incremental capital has a direct effect on the optimal time at which to expand lumpy capital.

Intuitively, increasing the cost of incremental capital investment decreases the value of the project once it has been initiated, which delays the initiation of the project. Given that the postexercise firm value is linear in $x$ and that there is zero cash flow before the exercise, a standard argument allows us to solve for $\bar{x}$ in closed form; see, for example, Dixit and Pindyck (1994). Guessing the functional form of $V(x, 0)$ and applying the value-matching and smooth-pasting conditions given in (8) and (9) shows that $\bar{x}$ solves the following equation

$$
\underbrace{(\eta-1)\left(\frac{1-\theta g\left(i^{*}\left(x, k_{\mathrm{b}}\right)\right)}{r-i^{*}\left(x, k_{\mathrm{b}}\right)}\right) \bar{x} k_{\mathrm{b}}}_{\begin{array}{c}
\text { marginal cost } \\
\text { of delaying investment }
\end{array}}=\underbrace{\eta p k_{\mathrm{b}}}_{\begin{array}{c}
\text { marginal benefit } \\
\text { of delaying } \\
\text { investment }
\end{array}}
$$

where $\eta>1$ is a constant that depends on $r, i_{\max }$, and $\sigma$. Equation (12) states that at the optimal investment threshold $\bar{x}$, the marginal cost of delaying investment in lumpy capital must equal the marginal benefit. More specifically, the left-hand side is the marginal cost of delay due to postponing the increase in cash flows that results from investment in new lumpy capital. The right-hand side is the marginal benefit of delay that results from postponing the expenditure of $p k_{\mathrm{b}}$.

The effect of an increase in incremental investment $\operatorname{costs} \theta$ on the optimal lumpy capital investment threshold $\bar{x}$ is determined by how such an increase affects the marginal benefit and cost of delaying investment. Differentiating both sides of (12) with respect to $\theta$ shows the following:

$$
\frac{\partial \bar{x}}{\partial \theta}=\bar{x}\left(\frac{1-\theta g\left(i^{*}\left(x, k_{\mathrm{b}}\right)\right)}{r-i^{*}\left(x, k_{\mathrm{b}}\right)}\right)^{-1}\left(\frac{g\left(i^{*}\left(x, k_{\mathrm{b}}\right)\right)}{r-i^{*}\left(x, k_{\mathrm{b}}\right)}\right) \geq 0 .
$$

In words, increasing the cost of investing in incremental capital decreases the marginal cost of delay because it lowers the present value of the cash flows that result from investment in lumpy capital. At the same time, such an increase does not affect the marginal benefit of delay. As a result, increasing $\theta$ delays investment in lumpy capital. Formally, we have the following result. 
Proposition 2. Suppose that $k_{\mathrm{s}}=0$. An increase in $\theta$ increases the optimal exercise threshold $\bar{x}$.

Note that Proposition 2 holds for any weakly convex $g(i)$ such that the result is not driven by the shape of the cost of incremental capital. Rather, the driving force behind the above result is that the cost only affects the optimal accumulation of incremental capital, and hence the value of the firm, after the exercise of the real option. As we show below, once this cost directly affects the pre-exercise accumulation of incremental capital, the sign of the effect of an increase in $\theta$ on the optimal exercise boundary $\bar{x}$ can change.

\subsection{Linear Incremental Investment Cost}

In this section, we analyze the case in which a firm begins with a positive amount of lumpy capital, $k_{s}>0$, and pays a linear cost to add incremental capital, $g(i)=i$. We analyze this case for two reasons. First, this specification has a natural interpretation. When $g(i)=i, \theta$ represents the unit price of $x$ scaled by $k$. Second, linear investment costs allow for closed-form solutions that yield transparent results. As we show in the subsequent analysis, the intuition and results from the linear cost case carry over to specifications with richer cost functions.

Given the linear cost function, the optimal investment rate $i^{*}$ is either 0 or $i_{\max }$. Indeed, the marginal benefit of an additional unit of incremental capital is $\frac{\partial}{\partial x} V(x, k)$ such that if the marginal cost of this unit is constant, it is optimal to invest either as much as possible (i.e., set $i=i_{\max }$ ) or as little as possible (i.e., set $i=0$ ). After the exercise of the option to expand lumpy capital, the marginal benefit of incremental capital is expressed as follows:

$$
\frac{\partial}{\partial x} V\left(x, k_{\mathrm{b}}\right)=\frac{1-\theta i}{r-i} k_{\mathrm{b}}
$$

Thus, if $\theta<\frac{1}{r}$, it is optimal to set $i=i_{\max }$. This condition is independent of the amount of incremental capital. The option to increase lumpy capital provides an additional benefit from investing in incremental capital. Consequently, it must also hold that it is optimal to select $i_{\max }$ before the exercise of the option to expand lumpy capital whenever $\theta<\frac{1}{r}$. Thus, it is natural to consider two separate cases within the set of feasible parameters. First, we analyze the problem when $\theta<\frac{1}{r}$ and thus $i\left(x, k_{\mathrm{s}}\right)=i\left(x, k_{\mathrm{b}}\right)=i_{\max }$. Next, we analyze the problem when $\theta>\frac{1}{r}$. In this case, it is optimal to set $i\left(x, k_{\mathrm{b}}\right)=0$. However, before the exercise of the option to expand lumpy 
capital, the additional benefit of incremental capital implied by the option to expand means that for some regions of $x$, it is optimal to set $i\left(x, k_{\mathrm{s}}\right)=i_{\max }$.

First consider the case in which $\theta<\frac{1}{r}$. In this case, the optimal incremental capital investment is $i_{\text {max }}$ regardless of the current stock of $k$. It follows that the cash flow per unit of $k$ and cash flow growth are the same both before and after the firm exercises the option to increase $k$. As in the previous section, applying the value-matching and smooth-pasting conditions shows that the optimal exercise boundary $\bar{x}$ is given by equating the marginal cost and the marginal benefit of delaying investment

$$
\underbrace{(\eta-1)\left(\frac{1-\theta i_{\max }}{r-i_{\max }}\right)\left(k_{\mathrm{b}}-k_{\mathrm{s}}\right) \bar{x}}_{\begin{array}{c}
\text { marginal cost } \\
\text { of delaying investment }
\end{array}}=\underbrace{\eta p\left(k_{\mathrm{b}}-k_{\mathrm{s}}\right)}_{\begin{array}{c}
\text { marginal benefit } \\
\text { of delaying } \\
\text { investment }
\end{array}} .
$$

The left-hand side of Equation (13) is the marginal cost of delaying lumpy capital investment and, as is also the case in Equation (12), is proportional to the increment $\left(1-\theta i_{\max }\right)\left(k_{\mathrm{b}}-k_{\mathrm{s}}\right) \bar{x}$ in the level of cash flows that results from investing in lumpy capital. An increase in the cost of incremental capital investment $\theta$ leads to decreases in cash flow both before and after lumpy capital investment proportional to the stock of lumpy capital and therefore decreases the cash flows that result from lumpy capital investment. As a result, an increase in $\theta$ lowers the marginal cost of delaying investment:

$$
\frac{\partial}{\partial \theta}\left[(\eta-1)\left(\frac{1-\theta i_{\max }}{r-i_{\max }}\right)\left(k_{\mathrm{b}}-k_{\mathrm{s}}\right)\right]=-(\eta-1)\left(\frac{i_{\max }}{r-i_{\max }}\right)\left(k_{\mathrm{b}}-k_{\mathrm{s}}\right)<0 .
$$

At the same time, such an increase in $\theta$ does not affect the marginal benefit of delaying investment $\eta p\left(k_{\mathrm{b}}-k_{\mathrm{s}}\right)$. In sum, an increase in $\theta$ lowers the marginal cost of delaying investment in lumpy capital and does not affect the marginal benefit, and it therefore increases $\bar{x}$ and delays investment in lumpy capital.

Now consider the case in which $\theta>\frac{1}{r}$. In this case, as we argue above, it is not optimal to invest in incremental capital after the exercise of the real option. Prior to the exercise of the lumpy capital growth option, incremental capital growth increases both current cash flows and the value of the option to expand lumpy capital. When the current stock of incremental capital is small, the likelihood that it will grow to the point where it is optimal to expand lumpy capital is so remote 
that investment in incremental capital is not optimal. However, when $x$ is sufficiently close to $\bar{x}$, an increase in incremental capital leads to a substantial increase in the value of the option to expand lumpy capital, and it is optimal to set $i^{*}\left(x, k_{\mathrm{S}}\right)=i_{\max }$ if $\theta \leq \frac{k_{\mathrm{b}}}{k_{\mathrm{s}}}\left(\frac{1}{r}\right)$. The intuition is that close to the exercise boundary $\bar{x}$, the cost of investment in incremental capital is proportional to $k_{\mathrm{s}}$, but the benefit is proportional to $k_{\mathrm{b}}$. In this case, the optimal exercise boundary $\bar{x}$ satisfies the following optimality condition

$$
\underbrace{(\eta-1)\left(\frac{k_{b}}{r}-\left(\frac{1-\theta i_{\max }}{r-i_{\max }}-m(\bar{x})\right) k_{s}\right)}_{\begin{array}{c}
\text { marginal cost } \\
\text { of delaying investment }
\end{array}} \bar{x}=\underbrace{\eta p\left(k_{\mathrm{b}}-k_{\mathrm{s}}\right)}_{\begin{array}{c}
\text { marginal benefit } \\
\text { of delaying investment }
\end{array}}
$$

where $m(\bar{x})$ is a term that accounts for the fact that prior to investment in lumpy capital, the firm does not invest in incremental capital when $x$ is small.

Comparing Equation (15) to Equation (13), we see that the key difference is in the marginal cost of delaying investment in lumpy capital. This term now accounts for the fact that net cash flows per unit of capital change from $\left(1-\theta i_{\max }\right) x$ to $x$ and cash flow growth changes from $i_{\max }$ to zero. Both of these changes occur because the optimal investment rate in incremental capital changes from $i_{\max }$ to zero at the moment the firm invests in lumpy capital.

Again, the effect of an increase in $\theta$ on the threshold $\bar{x}$ operates through the marginal cost of delaying investment. Now, an increase in $\theta$ increases the cash flows that result from investment in lumpy capital because such an increase does not affect the cash flow after the investment in new lumpy capital, and it decreases the cash flow beforehand. One can show that the effect that $\theta$ has on $m(x)$ is small, and thus an increase in $\theta$ leads to an increase in the marginal cost of delaying investment

$$
\frac{\partial}{\partial \theta}\left[(\eta-1)\left(\frac{k_{b}}{r}-\left(\frac{1-\theta i_{\max }}{r-i_{\max }}-m(\bar{x})\right) k_{s}\right)\right]=\left(\frac{i_{\max }}{r-i_{\max }}+\frac{\partial}{\partial \theta} m(\bar{x})\right) k_{s}>0 .
$$

Thus, when $\theta \geq 1 / r$, an increase in $\theta$ decreases the marginal benefit of delaying investment and lowers $\bar{x}$. We formalize these results in the proposition below.

Proposition 3. Suppose that $g(i)=i$. The optimal investment threshold increases with $\theta$ if $\theta$ is low and decreases with $\theta$ if $\theta$ is high. That is, $\frac{\partial \bar{x}}{\partial \theta}>0$ if $\theta<\frac{1}{r}$ and $\frac{\partial \bar{x}}{\partial \theta} \leq 0$ if $\frac{1}{r}<\theta<\frac{1}{r}\left(\frac{k_{\mathrm{b}}}{k_{\mathrm{s}}}\right)$. 
The important new result here lies in the effect of the cost of investing in incremental capital $\theta$ on the optimal threshold at which to invest in lumpy capital. Intuitively, increasing the cost of incremental capital should decrease the value of investment in lumpy capital and hence raise the threshold $\bar{x}$. However, this intuition ignores the effect that the increase in lumpy capital has on the optimal investment policy in incremental capital. If the cost of incremental capital is relatively

low, then it is optimal to invest in $x$ at the maximum rate both before and after exercising the real option. In this case, an increase in the cost of incremental capital would have a larger marginal effect on post-expansion firm value than on pre-expansion firm value, and the preceding intuition is correct. We illustrate this intuition in Figure 1 Panel A. Alternatively, if the cost of incremental capital is relatively high, then it is optimal to invest in $x$ at the maximum rate before exercising the real option and to forgo investment afterward. In this case, an increase in the cost of incremental capital has a greater marginal effect on the pre-investment firm than on the post-investment firm. In other words, further increasing the cost of incremental capital decreases the value of the preinvestment firm and does not change the post-investment value of the firm. Hence, the optimal investment threshold decreases. We illustrate this intuition in Figure 1, Panel B.

The intuition we outline above underlies the deep mechanism of our model. Although the linear specification of the cost of incremental capital growth is both tractable and economically relevant, the question remains whether this mechanism is generalizable to richer specifications of this cost function. In the next section, we demonstrate that this effect persists even when the cost function is strictly convex and the optimal investment rate in incremental capital is interior.

\subsection{Convex Adjustment Costs}

Our linear specification for the costs of investing in incremental capital in the preceding section has the advantage of allowing closed-form solutions and analytical comparative statics. However, it is somewhat restrictive in that it implies that the investment rate in incremental capital is either maximal or zero. One may then be concerned that our results are an artifact of this feature of the model. To allay such concerns, in this section, we analyze the cost of investment that includes a convex adjustment cost and stipulates interior investment rates.

To illustrate the model's implications, we use a particular parameterization. Following He (2011), we use a risk-free rate of $r=5 \%$ and a standard deviation of productivity growth of $\sigma=0.25$. 
Panel A. Delayed investment

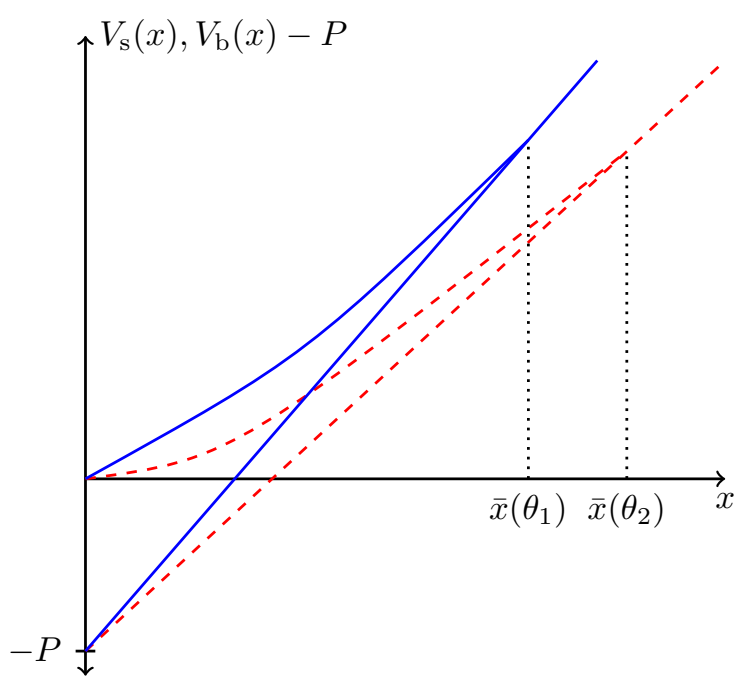

Panel B. Accelerated investment

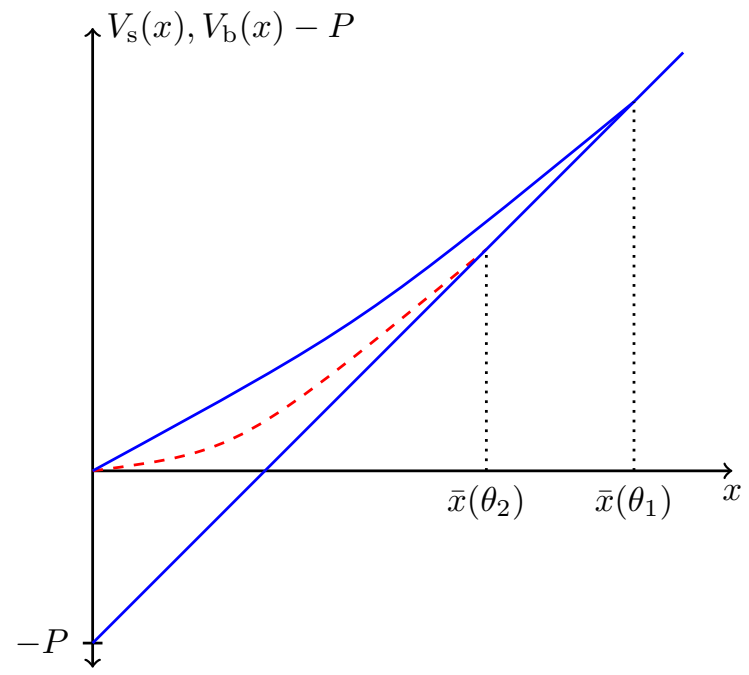

Figure 1. Effect of the cost of investment in incremental capital on the first-best investment threshold. This figure illustrates the optimal investment threshold for two possible costs of investment in incremental capital, $\theta_{1}<\theta_{2}$. In both panels, the solid curves represent the value functions for the pre-exercise (upper solid curve) and post-exercise (lower solid curve) firm when $\theta=\theta_{1}$. The dashed curves represent the value functions for the pre-exercise (upper dashed curve) and post-exercise (lower dashed curve) firm when $\theta=\theta_{2}$. In Panel A, which illustrates a low cost of investment in productivity growth, increasing the cost from $\theta_{1}$ to $\theta_{2}$ decreases both the pre- and post-exercise firm values but has a larger effect on the latter. Thus, such an increase leads to an increase in $\bar{x}$. In Panel $\mathrm{B}$, which illustrates a high cost of investment in incremental capital, increasing the cost from $\theta_{1}$ to $\theta_{2}$ decreases the pre-exercise firm value but has no effect on the post-exercise firm value, as the post-exercise investment in incremental capital is zero. Thus, such an increase in $\theta$ leads to a decrease in $\bar{x}$. 
The maximum growth rate of incremental capital needs to be less than the risk-free rate to ensure finite valuations for all levels of $\theta$. We choose an upper bound on the growth rate of $x$ of $i_{\max }=3 \%$. This choice is below that in He (2011) and reflects the fact that in our model, the growth rate of productivity is bounded below by 0 because of the non-negativity of incremental capital investment and the multiplicative specification for the effect of incremental capital on productivity. The cost of investment in incremental capital includes a quadratic adjustment term, $g(i)=i+\frac{1}{2} \psi\left(\frac{i}{i_{\max }-i}\right)^{2}$, where we use $\psi=0.05$. The denominator of the adjustment cost, $i_{\max }-i$, ensures that the marginal cost of incremental investment is infinite at $i_{\max }$ so that non-zero investment rates are interior. Lumpy capital increases at the time of investment from $k_{\mathrm{s}}=1$ to $k_{\mathrm{b}}=2$ at cost $p=10$ per unit of new capital.

Using these parameter values, Figure 2 Panel A presents the effects of the cost of incremental capital on the investment threshold. As is the case with linear investment costs, an increase in the cost of incremental capital measured by $\theta$ can lead to either an increase or decrease in the threshold to exercise the option to invest in lumpy capital. When the investment $\operatorname{cost} \theta$ is relatively low, an increase in $\theta$ leads to an increase in the investment threshold. If $\theta$ is somewhat higher, an increase in $\theta$ leads to a decrease in the investment threshold. The level of $\theta$ at which $\bar{x}$ starts to decrease is denoted by $\theta^{*}$. Figure 2, Panel $\mathrm{B}$ shows that introducing and increasing the convex adjustment cost expands the region in which $\bar{x}$ decreases with $\theta$. The figure plots the cutoff level $\theta^{*}$ for various levels of $\psi$, the parameter scaling the adjustment cost. As $\psi$ increases, $\theta^{*}$ decreases, which increases the parameter space in which $\bar{x}$ decreases.

Figure 2, Panel $\mathrm{C}$ shows the investment rates in incremental capital just before and after the exercise of the real option, $i^{*}\left(\bar{x}, k_{\mathrm{S}}\right)$ and $i^{*}\left(\bar{x}, k_{\mathrm{b}}\right)$, respectively. When $\theta$ is small enough and both $i^{*}\left(\bar{x}, k_{\mathrm{S}}\right)$ and $i^{*}\left(\bar{x}, k_{\mathrm{b}}\right)$ are high, $\bar{x}$ increases with $\theta$. Consistent with the intuition in the linear cost case, for $\bar{x}$ to decrease with $\theta$, the investment rate in incremental capital must drop at the moment of investment. If this drop is sufficiently large, then increasing $\theta$ decreases pre-investment firm value more than post-investment firm value, and the optimal $\bar{x}$ decreases. This occurs when $\theta$ is large enough to generate a non-monotonic relation between $\bar{x}$ and $\theta$. Finally, Figure 2, Panel D demonstrates that the accumulation of incremental capital intensifies as $x$ approaches the exercise threshold $\bar{x}$ because the growth option becomes more valuable closer to $\bar{x}$, increasing the benefits of investing in incremental capital. 
Panel A
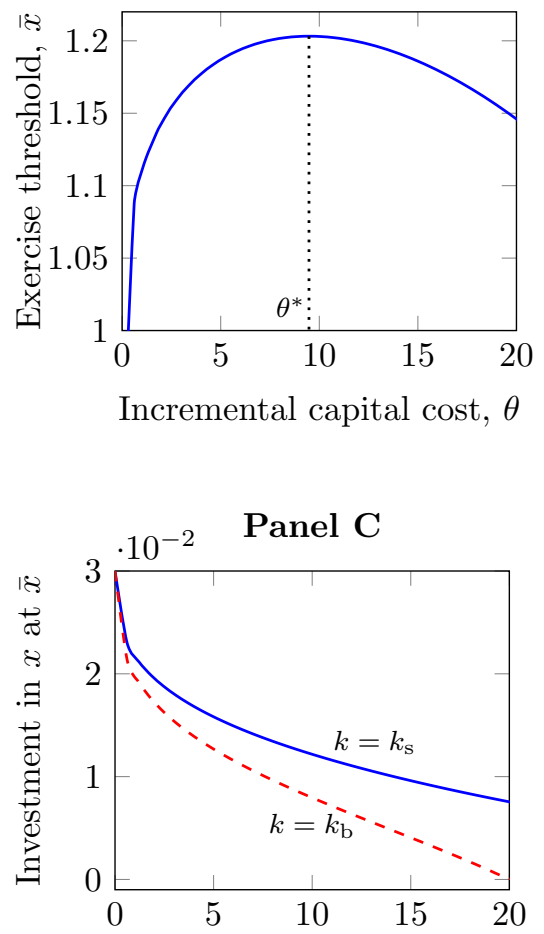

Incremental capital cost, $\theta$
Panel B

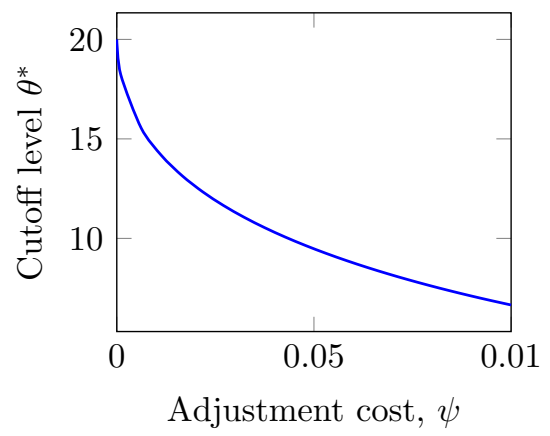

Panel D

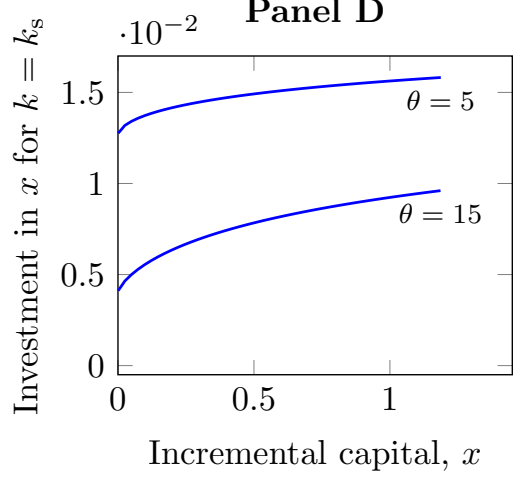

Figure 2. Adjustment costs of incremental capital and the investment threshold $\bar{x}$. Panel A plots the real option exercise threshold $\bar{x}$ for different levels of $\theta$. The cutoff level $\theta^{*}$ separates the regions of delayed and accelerated investment in lumpy capital. Panel B plots $\theta^{*}$ for different levels of the adjustment costs of investing in incremental capital $\psi$. Panel $\mathrm{C}$ plots the investment rates in incremental capital at $\bar{x}$ before and after the exercise of the real options using solid and dashed curves, respectively. Panel D plots the investment rates in incremental capital as a function of $x$ for various values of $\theta$. The constant parameter values are $r=0.05, \sigma=0.25$, $i_{\text {max }}=0.03, \psi=0.05, k_{\mathrm{s}}=1, k_{\mathrm{b}}=2$, and $p=10$. 


\section{Investment in Incremental Capital and Moral Hazard}

The results of the previous section highlight a new relation between investment in a capital stock that can be adjusted smoothly, i.e., incremental capital, and one that can be adjusted only in a lumpy fashion, i.e., lumpy capital. Although it is perhaps counterintuitive that increasing the cost of accumulating incremental capital can accelerate the exercise of a real option to expand lumpy capital, this result depends on the cost being high. This calls into question whether the result is empirically relevant.

In this section, we extend the model to include moral hazard over investment in incremental capital. This extension is natural to consider for a variety of reasons. First, investment in incremental capital is difficult to observe in reality and is thus likely to be subject to moral hazard. Second, this moral hazard problem can increase the cost of incremental capital such that even when the real cost of incremental capital is low, the total cost to the investor is high. Third, variation in moral hazard provides an empirically relevant source of variation to study in the data. Indeed, two firms that operate in the same line of business can face identical direct costs of incremental capital accumulation yet substantially different amounts of moral hazard, leading to different investment patterns. Finally, moral hazard provides an endogenous link between volatility and the cost of incremental capital that overturns a classic result in real option theory.

\subsection{Moral Hazard Problem}

We consider the model setup that we described in Section 2.1 with one important difference. We now assume that although the investor can directly control the exercise of the real option to expand lumpy capital, she must contract with a manager to implement investment in incremental capital. We further assume that the investor does not observe depreciation shocks to incremental capital. The total cost of investment in incremental capital is the same as before, but because the investor cannot observe investment in incremental capital, the manager can divert funds allocated to cover these costs for her own consumption. The manager has CARA preferences over consumption and values a stream of consumption $\left\{\tilde{c}_{t}\right\}$ :

$$
E\left[\int_{0}^{\infty}-\frac{1}{\gamma} e^{-\gamma \tilde{c} t-r t} d t \mid\{i\}\right]
$$


where $\gamma$ is a measure of risk aversion. In addition, the manager can save and borrow at the risk-free rate $r$. We assume that the manager begins with zero savings.

We use the manager's coefficient of risk aversion $\gamma$ to measure the severity of the moral hazard problem because, as we show below, $\gamma$ scales the cost of providing the manager with incentives to implement a given incremental capital investment policy. In an extended version of our model that includes shocks to incremental capital that are observable to the investor, it can be shown that the degree of observability of the manager's effort works in the same way as the coefficient of the manager's risk aversion. The former measure of the moral hazard problem has some advantages in that it is easier to estimate and varies more between firms and across time. We use $\gamma$ as a measure of the severity of the moral hazard problem for its simplicity. Appendix C.2 presents the extension with partially observable shocks to incremental capital.

A contract consists of a compensation rule, a recommended investment rate in $X$, and a stopping time denoted by $\Pi=(c, i, \tau)$. The compensation rule $\left\{c_{t}\right\}_{t \geq 0}$ and recommended investment rate $\left\{i_{t}\right\}_{t \geq 0}$ are stochastic processes adapted to the filtration of public information, $\mathcal{F}_{t}$. For simplicity, we drop the subscript $t$ whenever we refer to the entire process of consumption or incremental investment. The investment policy $\tau$ is $\mathcal{F}_{t}$-stopping time, which dictates when the firm exercises the option to increase capital.

Given an initial outside option of the manager $w_{0}$, the investor solves the following problem:

$$
\max _{c, i, \tau} E\left[\int_{0}^{\infty} e^{-r t}\left(X_{t} K_{t}-\theta g\left(i_{t}\right) X_{t} K_{t}-c_{t}\right) d t-e^{-r \tau} P\right]
$$

such that

$$
i \in \underset{\tilde{i}}{\arg \max }\left\{\max _{\tilde{s}} E\left[\int_{t}^{\infty}-\frac{1}{\gamma} e^{-\gamma\left(c_{t}+\theta\left(g\left(i_{t}\right)-g\left(\tilde{i}_{t}\right)\right) X_{t} K_{t}-\tilde{s}_{t}\right)-r(s-t)} d s\right]\right\},
$$

where $s_{t}$ is the amount the manager adds to her savings at time $t$. The key difference between the investor's problem under no moral hazard given in Equation (4) and the problem given in Equation (17) is that in addition to the direct cost of investing in incremental capital, the investor must also compensate the manager. This compensation, $c_{t}$, must in turn provide the manager with incentives to choose the recommended investment rate $i$.

In Appendix A.1, we provide a detailed analysis of the solution to the optimal contracting prob- 
lem. The key outcome of that analysis for the current problem is that the manager's continuation utility (i.e., the present value of all future consumption at any point in time) must be sufficiently sensitive to innovations in $X$. This incentive compatibility condition in turn implies that the manager's compensation $c_{t}$ is risky. As the manager is risk-averse and the investor is risk-neutral, risk in $c_{t}$ causes a loss in the form of forgone risk-sharing benefits. This loss acts as an extra cost of investment in incremental capital. We solve for these costs in the closed form in Appendix A.1. This allows us to show that the solution to the investor's problem in Equation (17) is given by the solution to the following HJB equation:

$$
r V=\max _{i \in\left[0, i_{\max }\right]}\left\{x k(1-\theta g(i))+i x \frac{\partial V}{\partial x}+\frac{1}{2} \sigma^{2} x^{2} \frac{\partial^{2} V}{\partial x^{2}}-\rho(i, x, k)\right\}
$$

where

$$
\rho(i, x, k)=\frac{1}{2} \mathbb{1}(i>0) \gamma r\left(\theta \sigma g^{\prime}(i) x k\right)^{2}
$$

represents the incentive cost of investment in $X$. The HJB equation in (19) is identical to that in Equation (5) up to the additional cost given by $\rho$. The optimal investment policy is again defined by a threshold $\bar{x}$ at which it is optimal for the investor to increase lumpy capital. The value function must satisfy the same standard value-matching and smooth-pasting conditions as in the first-best case. We verify that this approach indeed yields the optimal contract in the proof of the following proposition in Appendix B.

Proposition 4. The optimal contract under moral hazard is given by the solution to Equation (19) with the boundary conditions in Equations (8)-(10).

\subsection{Incentives and Real Option Exercise: Linear Investment Cost}

In this section, we return to the linear investment cost specification we study in Section 3.2. As in that section, we consider $g(i)=i$. We also restrict attention to $\theta \leq \frac{1}{r}$. Recall that without moral hazard, this restriction implies that the firm always sets $i=i_{\max }$ and that an increase in the cost of incremental capital thus delays the exercise of the real option to expand lumpy capital. With moral hazard, investment in incremental capital entails an additional incentive cost that is increasing and convex in $x$. As such, it is not always optimal to invest in incremental capital at the 
maximal rate. This implies that even when the direct cost of investment in $x$ is low, an increase in the total cost via an increase in the severity of the moral hazard problem $\gamma$ can decrease the exercise threshold $\bar{x}$.

We first consider the optimal investment rate after the exercise of the option to expand lumpy capital. Given that the total cost of investment in incremental capital is convex in $x$, we hypothesize that there is a threshold $x_{\mathrm{b}}^{*}$ below which the contract recommends maximal investment and above which it recommends zero investment:

$$
i^{*}\left(x, k_{\mathrm{b}}\right)= \begin{cases}i_{\max } & \text { if } x \leq x_{\mathrm{b}}^{*} \\ 0 & \text { otherwise }\end{cases}
$$

The explicit solutions for $x_{\mathrm{b}}^{*}$ are provided in Appendix A.2. This control satisfies Equation (19) and is therefore optimal.

Before exercising the option to expand lumpy capital, it is optimal to implement maximal investment in incremental capital as long as the price $p$ of the new lumpy capital is sufficiently small. Thus, there are two natural cases to consider. In the first case, investment in lumpy capital leads to no change in the current rate of investment in incremental capital - that is, $\bar{x} \leq x_{\mathrm{b}}^{*}$. In the second case, when investment in lumpy capital leads to a decrease in the current rate of investment in incremental capital - that is, $\bar{x} \geq x_{\mathrm{b}}^{*}$. In the appendix, we show that $\bar{x} \leq x_{\mathrm{b}}^{*}$ if and only if $\gamma \leq \gamma_{1}$ for some constant $\gamma_{1}$. Intuitively, when $\gamma$ is small, incentives are relatively inexpensive, and a high rate of incremental capital investment will be optimal even after the exercise of the option to invest in lumpy capital.

When investment in lumpy capital leads to no change in the current rate of investment in incremental capital, we can combine the value-matching and smooth-pasting conditions to obtain

$$
\underbrace{(\eta-1)\left(\frac{1-\theta i_{\max }}{r-i_{\max }}\right)\left(k_{\mathrm{b}}-k_{\mathrm{s}}\right) \bar{x}}_{\text {of delaying increase in cash flow }}=
$$

$$
\underbrace{\eta p\left(k_{\mathrm{b}}-k_{\mathrm{s}}\right)}_{\begin{array}{c}
\text { marginal benefit } \\
\text { of delaying } \\
\text { investment expenditure }
\end{array}}+\underbrace{(\eta-2)\left(\frac{\gamma r(\theta \sigma)^{2}}{2\left(r-2 i_{\text {max }}-\sigma^{2}\right)}\right)\left(k_{\mathrm{b}}^{2}-k_{\mathrm{s}}^{2}\right) \bar{x}^{2}}_{\begin{array}{c}
\text { marginal benefit of delaying increase } \\
\text { in incentive costs }
\end{array}} .
$$


Comparing Equation (22) with Equation (13), we see that incentive costs, represented by the second term on the right-hand side of Equation (22), create an additional marginal effect of delaying investment in lumpy capital. In the Appendix, we show that $\eta-2$ and $r-2 i_{\max }-\sigma^{2}$ have the same sign, and thus the incentive cost term is always positive and represents a marginal benefit from delaying investment. In other words, investing in lumpy capital increases the flow of incentive costs given the optimal incremental investment policy. This intuition follows the standard narrative in the literature: increasing moral hazard decreases the net return of new capital and therefore curtails investment. As a result, increasing $\gamma$, i.e., the severity of the moral hazard problem, increases the marginal benefit of delay that is due to incentive costs and thus raises $\bar{x}$ and delays investment in lumpy capital.

When investment in lumpy capital leads to a decrease in the current rate of investment in incremental capital, the flow of incentive costs decreases once the new lumpy capital is installed. In this case, we can combine the value-matching and smooth-pasting conditions to obtain

$$
\begin{aligned}
& \underbrace{(\eta-1)\left(\frac{1-\theta i_{\max }}{r-i_{\max }}\right)\left(d_{1}(\bar{x}) k_{\mathrm{b}}-k_{\mathrm{s}}\right) \bar{x}}_{\text {of delaying increase in cash flow }}= \\
& \underbrace{\eta p\left(k_{\mathrm{b}}-k_{\mathrm{s}}\right)}_{\begin{array}{c}
\text { marginal benefit } \\
\text { of delaying } \\
\text { nvestment expenditure }
\end{array}}+\underbrace{(\eta-2)\left(\frac{\gamma r(\theta \sigma)^{2}}{2\left(r-2 i_{\max }-\sigma^{2}\right)}\right)\left(d_{2}(\bar{x}) k_{\mathrm{b}}^{2}-k_{\mathrm{s}}^{2}\right) \bar{x}^{2}}_{\text {marginal effect of delaying change in incentive costs }},
\end{aligned}
$$

where $d_{1}(x)$ and $d_{2}(x)$ account for the fact that for small $x$, the firm will invest in incremental capital even after the exercise of the lumpy capital expansion option. ${ }^{7}$ It is possible to show that $d_{2}(\bar{x}) k_{\mathrm{b}}^{2}-k_{\mathrm{s}}^{2}>0$ if and only if $\gamma<\gamma_{2}$ for some constant $\gamma_{2}$. Thererfore, when $\gamma<\gamma_{2}$, the marginal effect of delaying investment in lumpy capital that is due to the change in incentive cost represents a marginal benefit of delay. This effect is due to the fact that although the flow of incentive costs decreases at the moment of investment, they increases if $x$ falls below $x_{\mathrm{b}}^{*}$. In this case, an increase in $\gamma$ delays investment for the same intuition we give above.

When $\gamma>\gamma_{2}$, the marginal effect of delaying investment in lumpy capital due to the change in incentive costs represents a marginal cost of delay. As a result, increasing the moral hazard

\footnotetext{
${ }^{7}$ The explicit solutions for $d_{1}(x)$ and $d_{2}(x)$ and are given in Appendix A.2.
} 
problem by increasing $\gamma$ increases the marginal cost of delaying investment in lumpy capital and lowers the investment threshold $\bar{x}$. In other words, investing in lumpy capital leads to a decrease in the flow of incentive costs, which makes investment more attractive. This effect is missing from the standard intuition for the effect of moral hazard on investment because that intuition relies on incentive costs remaining proportionally constant in capital under the optimal contract.

Proposition 5. Suppose that $\theta \leq \frac{1}{r}$ and $g(i)=i$. The optimal exercise threshold increases with the severity of the moral hazard problem when $\gamma$ is small and decreases when $\gamma$ is large. That is, there exists $\gamma_{2}<\infty$ such that $\frac{\partial \bar{x}}{\partial \gamma}<0$ if and only if $\gamma>\gamma_{2}$.

The explicit expression for $\gamma_{2}$ is given in the proof of Proposition 5. It is worth noting that while $\gamma_{2}$ is finite, it need not be positive. However, $\gamma_{2}>0$ whenever $k_{\mathrm{b}}$ is large relative to $k_{\mathrm{s}}$ or $i_{\text {max }}$ is small relative to $r$.

Proposition 5 gives the effect of moral hazard on investment in the case of a marginal increase in the severity of the moral hazard problem. The effect of an increase in $\gamma$ on $\bar{x}$ operates through a similar mechanism as the comparative static of $\bar{x}$ with respect to the $\theta$ determined in Section 3.2. In both cases, increasing the cost of investment in incremental capital (either direct or incentive costs) changes the optimal investment policy after the exercise of the real option, which can increase the benefit of expanding lumpy capital and lower the exercise threshold for the real option. In this sense, moral hazard amplifies the effect of incremental investment costs on the optimal exercise threshold.

In addition to amplifying the mechanism through which incremental investment costs affect the optimal lumpy investment policy, moral hazard can also cause the investment threshold $\bar{x}$ to fall below what it would have been without moral hazard, which we call $\bar{x}^{\mathrm{FB}}$. Intuitively, the investment threshold does not depend on $\gamma$ when there is no moral hazard problem, whereas an increase in $\gamma$ decreases $\bar{x}$ when there is moral hazard. This result stands in stark contrast to the literature, which has shown that moral hazard typically leads to underinvestment. In our model, moral hazard erodes option value and can lead to a form of overinvestment in that investors optimally exercise a growth option at a lower threshold than they would if there were no moral hazard problem. The following proposition formally states this result.

Proposition 6. Suppose that $\theta \leq \frac{1}{r}$ and $g(i)=i$. The investment threshold under moral hazard 
is below that of the first-best case if and only if the moral hazard problem is severe. That is, there exists $\gamma_{3}<\infty$ such that $\bar{x}<\bar{x}^{\mathrm{FB}}$ if and only if $\gamma>\gamma_{3}$.

\subsection{Volatility and Real Option Exercise}

A well-known finding in the real options literature is that an increase in volatility increases the value of the option to wait and thus increases the optimal exercise threshold. Without moral hazard, this result also applies to our model. However, moral hazard creates an endogenous link between volatility and the cost of investment in incremental capital through the incentive cost term $\rho$. Keeping everything else constant, incentive costs increase when volatility is high because the manager needs to be compensated for risk exposure. Increasing volatility thus has two effects in our model. First, increasing volatility increases the value of the option to wait to invest, as in a standard real options model. This effect operates through the dependence of Equations (22) and (23) on the term $\eta$. Second, when $\gamma \geq \gamma_{2}$, incentive costs create an additional cost of delaying invest in lumpy capital relative to the first-best case. In this case, increasing volatility amplifies the effect that incentive costs have on the marginal cost of delaying investment in lumpy capital. When incentive costs are sufficiently large, i.e., when $\gamma \geq \gamma_{4}$ for some constant $\gamma_{4}$, the second effect dominates the first and an increase in volatility accelerates investment. We formalize this intuition in the following proposition.

Proposition 7. Suppose that $\theta \leq \frac{1}{r}, g(i)=i$, and $r>2 i_{\max }+\sigma^{2}$. The optimal exercise boundary $\bar{x}$ decreases with volatility $\sigma$ when $\gamma$ is large. That is, there exists $\gamma_{4}<\infty$ such that $\frac{\partial \bar{x}}{\partial \sigma}<0$ for $\gamma>\gamma_{4}$

\subsection{Incentives with Convex Adjustment Costs}

In this section, we generalize the cost of investing in incremental capital to a convex function that includes direct-cost and adjustment-cost terms. As in Section 3.3, we assume that the cost

function takes the following form: $g(i)=i+\frac{1}{2} \psi\left(\frac{i}{i_{\max }-i}\right)^{2}$. This generalization allows us to analyze investment rates $i$ away from the corner $i_{\max }$ and verify the robustness of the tractable linear model of the preceding sections. In addition, the added flexibility enables a more convincing quantitative analysis than would be possible with simple linear investment costs. This allows us to evaluate the 

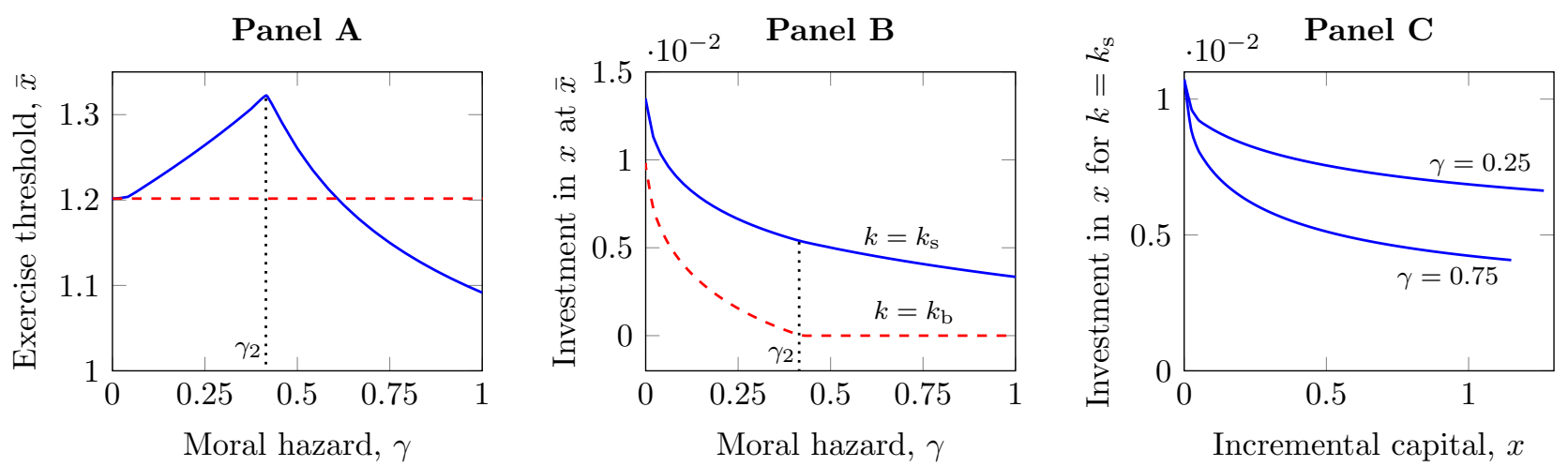

Figure 3. Moral hazard and optimal investment. Panel A plots the real option exercise threshold $\bar{x}$ as a function of the severity of the moral hazard problem $\gamma$ (the dashed line represents the first-best level for $\gamma=0$ ). Panel B plots the investment rates in incremental capital at $\bar{x}$ before and after the exercise of the real options using solid and dashed curves, respectively. Panel C plots the investment rates in incremental capital as a function of $x$ for various values of $\gamma$. The constant parameter values are $r=0.05, \sigma=0.25, i_{\max }=0.03, \theta=8, \psi=0.05, k_{\mathrm{s}}=1, k_{\mathrm{b}}=2$, and $p=10$.

relevance of the incentive costs for investment behavior.

We solve the model numerically using the parameter values introduced in Section 3.3. Figure 3 Panel A illustrates the effect of moral hazard on the exercise threshold. As is the case with linear costs (see Proposition 5), an increase in the severity of the moral hazard problem can lead to either an increase or decrease in the investment threshold. When the moral hazard problem measured by $\gamma$ is relatively low, then an increase in $\gamma$ leads to an increase in the investment threshold and underinvestment relative to the first-best case. If $\gamma$ is somewhat higher, an increase in $\gamma$ leads to a decrease in the investment threshold. Consistent with the result in the case of linear cost (see Proposition 6), if $\gamma$ is sufficiently high, the investment threshold is below that of the first-best case.

The incentive cost in Equation (20) generates an additional cost of investing in incremental capital and thus decreases the rate of investment $i$ relative to the first-best case. Next, we show that the incentive cost also has an important qualitative effect on the rate of investment in incremental capital. In the model without moral hazard, the rate $i$ increases with $x$; see Figure 2, Panel D. This is a growth option effect in that the benefit of investing in $x$ increases as the value of the growth option increases closer to the exercise threshold. In the model with moral hazard, there is an opposing effect. As it becomes increasingly expensive to incentivize the manager as the amount of incremental capital increases, the incentive cost is convex in $x$. Figure 3, Panel C demonstrates that for our parameter values and various values of $\gamma$, the incentive cost effect dominates the real 


\begin{tabular}{cccc}
\hline & \multicolumn{3}{c}{$\begin{array}{c}\text { Cost of incremental } \\
\text { investment }\end{array}$} \\
$\bar{x} / \bar{x}^{\mathrm{FB}}$ & $\theta=6$ & $\theta=8$ & $\theta=10$ \\
\hline Adjustment cost & & & \\
$\psi=0.02$ & 1.10 & 1.20 & 1.16 \\
$\psi=0.05$ & 1.07 & 1.05 & 0.92 \\
$\psi=0.08$ & 1.06 & 0.93 & 0.88 \\
\hline
\end{tabular}

Table 1. Under- and overinvestment due to agency conflicts. This table reports the ratios of the investment thresholds under agency to the investment thresholds in the first-best case. Values above one represent underinvestment and those below one represent overinvestment. The constant parameter values are $r=0.05, \sigma=0.25, i_{\max }=0.03, \gamma=0.5, k_{\mathrm{s}}=1, k_{\mathrm{b}}=2$, and $p=10$.

option effect, and the investment rate $i$ decreases with $x$ as $x$ approaches the exercise threshold.

The last observation indicates the possibility that even in cases in which optimal investment in lumpy capital is at a lower threshold under moral hazard than under the first-best case, moral hazard may delay exercise when measured in time units as the rate of investment in $x$ decreases. We verify this by numerically analyzing the expected time to exercise the growth option under moral hazard and in the first-best case. We find that the expected exercise times are always consistent with the relation of the exercise threshold for our baseline parameter values (for brevity, the results are not reported here). That is, a lower threshold is always associated with an earlier time to invest. This implies that the effect of moral hazard on the exercise threshold must be relatively large and dominate the weakened growth in $x$.

Indeed, quantitatively, the under- and overinvestment effects on the exercise threshold are large. To assess the magnitudes in greater detail, we examine a range of costs of investing in incremental capital. For the other parameters of the model, we use the values specified in Section 3.3. Table 1 presents the ratios of the investment thresholds under moral hazard to the investment thresholds in the first-best case. Values above one represent underinvestment and values below one represent overinvestment. Overinvestment is associated with low efficiency of investment in incremental capital (high $\theta$ and $\psi$ ). The threshold under agency is $12 \%$ smaller than in the first-best case with high costs of investment in incremental capital of $\theta=10$ and $\psi=0.08$. Underinvestment tends to be the highest with low costs of investment in incremental capital. For instance, when $\theta=8$ and $\psi=0.02$, the investment threshold under agency exceeds the first-best case by $20 \%$.

We close this subsection by showing that the results discussed above do not depend on incre- 

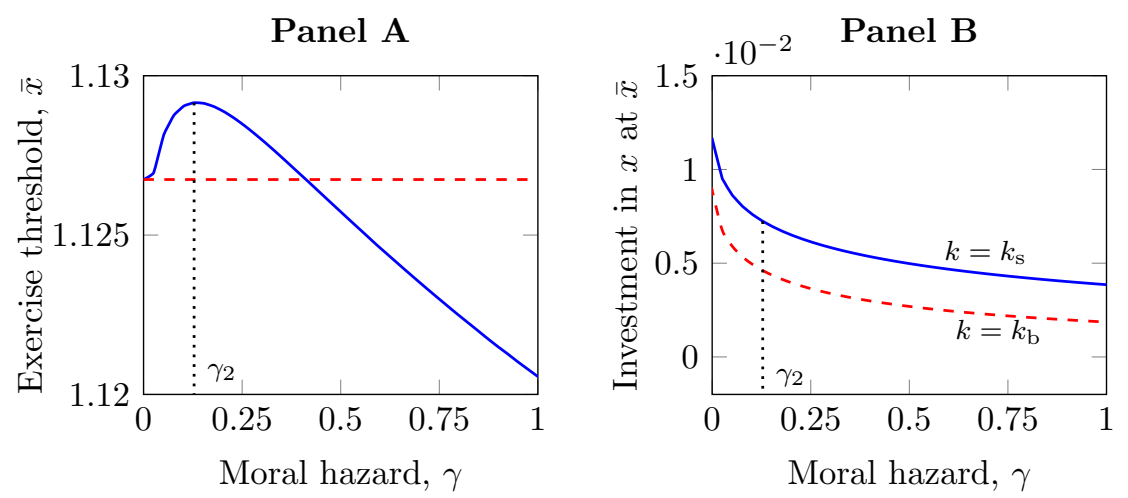

Figure 4. Moral hazard and optimal investment with interior incremental investment. Panel A plots the real option exercise threshold $\bar{x}$ as a function of the severity of the moral hazard problem $\gamma$ (the dashed line represents the first-best level for $\gamma=0$ ). Panel B plots the investment rates in incremental capital at $\bar{x}$ before and after the exercise of the real options using solid and dashed curves, respectively. The constant parameter values are $r=0.05, \sigma=0.25, i_{\max }=0.03$, $\theta=8, \psi=0.1, k_{\mathrm{s}}=1, k_{\mathrm{b}}=2$, and $p=10$.

mental investment reaching zero. Note that the direct linear cost of investing in incremental capital introduces a fixed incentive cost for non-zero investment levels. ${ }^{8}$ This fixed cost can cause large firms to stop investing in $x$, as is the case in the example in Panel B of Figure 3. To eliminate the influence of zero investment, we remove the linear cost from $g(i)$ and consider $g(i)=\frac{1}{2} \psi\left(\frac{i}{i_{\max }-i}\right)^{2}$. We use the same parameter values as in Figure 3 except that $\psi$ is increased from 0.05 to 0.1 to compensate for the removal of the direct cost of investment. The results presented in Figure 4, Panel A show that the non-monotonic relation between $\bar{x}$ and $\gamma$ is also present in this case. Panel B shows that investment in incremental capital drops at $\bar{x}$ but remains positive. This suffices to generate accelerated investment in lumpy capital. Specifically, when $\gamma$ is large, lumpy investment creates a sufficiently large drop in the incentive cost to induce a lower threshold $\bar{x}$.

\section{Discussion and Empirical Predictions}

In this section, we discuss the empirical relevance of our model. We first discuss some examples of specific instances in which incentive costs could affect the timing of investment. Next, we discuss industrial settings in which the forces that we identified above should be particularly salient and

\footnotetext{
${ }^{8}$ Specifically, the incentive cost, which is given in Equation (20), is a function of $g^{\prime}(i)$, which has a constant term if $g(i)$ includes a linear component.
} 
provide empirical predictions to guide future work.

\subsection{Incremental and Lumpy Capital Investment in Practice}

In this section, we give practical examples of the types of investment that we consider in our model. One example of a capital stock that managers must often develop is organization capital, as in the literature founded by Prescott and Visscher (1980). In particular, our model applies to incremental investment in organization capital that increases the productivity of physical capital that can only be added in a lumpy fashion. A large body of literature demonstrates the importance of organization capital for firm outcomes and includes Atkeson and Kehoe (2005), Carlin, Chowdhry, and Garmaise (2012), and Lustig, Syverson, and Van Nieuwerburgh (2011). Our model demonstrates that the moral hazard associated with the smooth accumulation of organization capital has important implications for investment in lumpy physical capital (for example, factories).

To be concrete, consider gains in efficiency that are the result of process innovations, i.e., the organizational capital associated with the knowledge of how to best use physical capital. ${ }^{9}$ To accumulate this type of organizational capital, the manager makes a number of changes to the firm's manufacturing processes that increase the efficiency (i.e., the productivity) of the existing machinery - for example, co-locating all of the machinery needed to complete one unit of output within a factory (cellular manufacturing). Finding the particular production process that maximizes the efficiency of a given firm's capital requires the manager to experiment and learn. This on its own may not be related to the amount of physical capital installed at the firm; however, as implementing changes requires the manager to communicate them to the many levels of workers involved in production, it is clear that a variety of control costs will be incurred. In this way, a manager can treat a small firm as a laboratory in which to hone her knowledge. Then, any new factories can begin operations with the benefit of the firm's accumulated knowledge or organizational capital.

A second example of the type of investment that we consider in our model is internal investment in innovation, which we contrast with external and lumpy investment in innovation via acquisitions. Evidence documented by Cassiman and Veugelers (2006) suggests that in general, internal and external innovation are complements, which is consistent with our model. For example, consider Facebook's acquisition of Instagram in 2006. In this case, Facebook engaged in internal innovation

\footnotetext{
${ }^{9}$ See, for example, the lean production process described by Shah and Ward (2007).
} 
by improving its own product to grow its user base and deepen the network of connections therein. Facebook then purchased Instagram, which increased users. The incremental revenue from new users from Instagram was magnified by the depth and size of Facebook's existing network. However, the question remains as to why Facebook purchased Instagram at such an early stage in Instagram's development rather than internally growing its own network of users and waiting to acquire Instagram. While anti-competitive forces may have played a role, our results provide an alternative explanation. At the point of acquisition, Facebook had developed to a point at which further internal innovation and growth carried significant incentive costs, which eroded its option value to wait to acquire Instagram and thus hastened its acquisition.

Facebook's acquisition of Instagram is one example of numerous instances of external innovation acquisitions by mature firms in the information technology industry. Many such acquisitions are surprising to market observers. In light of our model, the agency conflicts inherent in mature technology firms and the resulting high incentive costs of internal innovation make early external innovation acquisitions optimal.

\subsection{Empirical Predictions}

We now discuss the empirical implications of our model. As in the first setting discussed above, lumpy capital can refer to larger physical capital investments; in such cases, incremental capital corresponds to organization capital. Alternatively, as in the second setting discussed above, lumpy capital can refer to external acquisitions and incremental capital to internal growth.

We begin with two direct implications of our main results:

Prediction 1. Lumpy investment is delayed (accelerated) in response to an increase in the cost of acquiring incremental capital for low (high) measures of the cost.

Prediction 2. Lumpy investment is delayed (accelerated) in response to an increase in incentive costs for low (high) measures of the cost.

One distinguishing feature between the models with and without moral hazard can be seen in Figures 3 Panel $\mathrm{C}$ and 2 Panel D. In these figures, we plot the relation between the investment rate in incremental capital and its current level. Without moral hazard, the investment rate in incremental capital increases. When moral hazard is included, the investment rate in incremental 
capital decreases. The reason for this difference is that moral hazard introduces an extra cost to the accumulation of incremental capital (the cost is quadratic in the level of incremental capital). When the moral hazard problem is severe, this cost dominates and the investment rate in incremental capital decreases. This leads to the following prediction:

Prediction 3. For firms with substantial (negligible) moral hazard, incremental capital growth is negatively (positively) correlated with the level of capital.

\section{Conclusion}

We present a model of investment in lumpy and incremental capital. In our model, physical capital productivity is determined by a stock of incremental capital. The owners of a firm can accumulate incremental capital subject to a cost. We show that this cost is naturally affected by the presence of managerial moral hazard. We find that the effect of the costs of accumulating incremental capital on the timing of real option exercise depends on the magnitude of these costs. When the cost of investing in incremental capital is low, for example, because the moral hazard problem is not severe, it is optimal to implement high investment in incremental capital, and an increase in the cost raises the threshold for exercising the real option. When the cost of accumulating incremental capital is high, the opposite effect is obtained. The finding that a manager's ability to shirk or divert cash flow can increase investment is new, and it provides an alternative to empirebuilding and managerial hubris-based explanations of overinvestment.

Our model can be extended and applied to specific contexts in the real option literature without agency conflicts (e.g., mergers and acquisitions, real estate development, IPOs, and venture capital financing). In each case, we have omitted important institutional details from the model for clarity. However, an examination of these details may provide interesting new results and implications. For example, in mergers and acquisitions, investment may depend on the productivity of both the bidding and target firms. An important feature of real estate development that may interact with the agency conflict is that investment typically requires time to build. Finally, in IPOs and venture capital financing, the manager may have private information that affects the value of the growth option. 


\section{References}

Abel, A. B., and J. C. Eberly. 1996. Optimal investment with costly reversibility. Review of Economic Studies 63:581-93.

Atkeson, A., and P. J. Kehoe. 2005. Modeling and measuring organization capital. Journal of Political Economy 113:1026-53.

Balsmeier, B., L. Fleming, and G. Manso. 2017. Independent boards and innovation. Journal of Financial Economics 123:536-57.

Biais, B., T. Mariotti, G. Plantin, and J. Rochet. 2007. Dynamic security design: Convergence to continuous time and asset pricing implications. Review of Economic Studies 74:345-90.

Biais, B., T. Mariotti, J. Rochet, and S. Villeneuve. 2010. Large risks, limited liability, and dynamic moral hazard. Econometrica 78:73-118.

Brennan, M. J., and E. S. Schwartz. 1985. Evaluating natural resource investments. Journal of Business 58:135-57.

Carlin, B. I., B. Chowdhry, and M. J. Garmaise. 2012. Investment in organization capital. Journal of Financial Intermediation 21:268-86.

Cassiman, B., and R. Veugelers. 2006. In search of complementarity in innovation strategy: Internal R\&D and external knowledge acquisition. Management Science 52:68-82.

Datta, S., M. Iskandar-Datta, and K. Raman. 2001. Executive compensation and corporate acquisition decisions. Journal of Finance 56:2299-336.

DeMarzo, P., and M. Fishman. 2007. Agency and optimal investment dynamics. Review of Financial Studies 20:151-88.

DeMarzo, P., M. Fishman, Z. He, and N. Wang. 2012. Dynamic agency and the q theory of investment. Journal of Finance 67:2295-340.

DeMarzo, P., and Y. Sannikov. 2006. Optimal security design and dynamic capital structure in a continuous-time agency model. Journal of Finance 61:2681-724. 
Dixit, A. K., and R. S. Pindyck. 1994. Investment under uncertainty. Princeton, NJ: Princeton University Press.

Grenadier, S., and N. Wang. 2005. Investment timing, agency, and information. Journal of Financial Economics 75:493-533.

Grenadier, S. R., and A. Malenko. 2011. Real options signaling games with applications to corporate finance. Review of Financial Studies 24:3993-4036.

Grenadier, S. R., A. Malenko, and N. Malenko. 2016. Timing decisions in organizations: Communication and authority in a dynamic environment. American Economic Review 106:2552-81.

Gryglewicz, S., B. Hartman-Glaser, and G. Zheng. 2018. Growth options, incentives, and pay-forperformance: Theory and evidence. Management Science forthcoming.

Harford, J., and K. Li. 2007. Decoupling CEO wealth and firm performance: The case of acquiring CEOs. Journal of Finance 62:917-49.

He, Z. 2009. Optimal executive compensation when firm size follows geometric Brownian motion. Review of Financial Studies 22:859-92.

- 2011. A model of dynamic compensation and capital structure. Journal of Financial Economics 100:351-66.

Holmstrom, B., and P. Milgrom. 1987. Aggregation and linearity in the provision of intertemporal incentives. Econometrica 303-28.

Huergo, E., and J. Jaumandreu. 2004. How does probability of innovation change with firm age? Small Business Economics 22:193-207.

Jensen, M. C. 1986. Agency costs of free cash flow, corporate finance, and takeovers. The American Economic Review 76:323-9.

Lustig, H., C. Syverson, and S. Van Nieuwerburgh. 2011. Technological change and the growing inequality in managerial compensation. Journal of Financial Economics 99:601-27.

Malenko, A. 2018. Optimal dynamic capital budgeting. Review of Economic Studies forthcoming. 
McDonald, R., and D. Siegel. 1986. The value of waiting to invest. The Quarterly Journal of Economics 101:707-27.

Philippon, T., and Y. Sannikov. 2007. Real options in a dynamic agency model, with applications to financial development, IPOs, and business risk. Working Paper.

Piskorski, T., and A. Tchistyi. 2010. Optimal mortgage design. Review of Financial Studies 23:3098-140.

—. 2011. Stochastic house appreciation and optimal mortgage lending. Review of Financial Studies 24:1407-46.

Prescott, E. C., and M. Visscher. 1980. Organization capital. Journal of Political Economy 88:44661.

Sannikov, Y. 2008. A continuous-time version of the principal-agent problem. Review of Economic Studies 75:957-84.

Shah, R., and P. T. Ward. 2007. Defining and developing measures of lean production. Journal of Operations Management 25:785-805.

Spear, S., and S. Srivastava. 1987. On repeated moral hazard with discounting. Review of Economic Studies 54:599-617.

Stein, J. C. 2003. Agency, information and corporate investment. Handbook of the Economics of Finance 1:111-65.

Strulovici, B., and M. Szydlowski. 2015. On the smoothness of value functions and the existence of optimal strategies in diffusion models. Journal of Economic Theory 159:1016-55.

Tirole, J. 2010. The theory of corporate finance. Princeton, NJ: Princeton University Press.

Titman, S., K. J. Wei, and F. Xie. 2004. Capital investments and stock returns. Journal of Financial and Quantitative Analysis 39:677-700.

Zhao, X. 2009. Technological innovation and acquisitions. Management Science 55:1170-83. 


\section{Appendix A: Solving the Moral Hazard Problem}

In this appendix, we provide the solution to the optimal contracting problem in Section 4 and the value functions used in Section 4.2.

\section{A.1 Optimal Contract}

Because the manager can privately save, the compensation specified by the contract $c_{t}$ need not be equal to the manager's consumption at time $t$. The manager's accumulated savings is denoted by $S_{t}$, her actual time $t$ consumption by $\tilde{c}_{t}$, and her incremental investment by $\tilde{i}_{t}$. Given a contract, the manager chooses a consumption and incremental investment plan to maximize her utility from the contract:

$$
W_{t}\left(\Pi,\left\{X_{s}, K_{s}\right\}_{s \leq t} ; \mathcal{S}\right)=\max _{\tilde{c}, \tilde{i}} E\left[\int_{t}^{\infty} e^{-r(s-t)} u\left(\tilde{c}_{s}\right) d s\right]
$$

where the manager's instantaneous utility is

$$
u(\tilde{c})=-\frac{1}{\gamma} e^{-\gamma \tilde{c}}
$$

such that $X_{t}, S_{t}$, and $K_{t}$ have the dynamics induced by consumption and the incremental investment plan $(\tilde{c}, \tilde{i})$ :

$$
\begin{aligned}
& d S_{s}=r S_{s} d s+\left(\tilde{c}_{s}-c_{s}+\theta(g(\tilde{i})-g(i)) X_{s} K_{s}\right) d s, \quad S_{t}=\mathcal{S}, \\
& d X_{s}=\tilde{i}_{s} X_{s} d s+\mathbb{1}\left(\tilde{i}_{s}>0\right) \sigma X_{s} d Z_{s}, \\
& K_{s}= \begin{cases}k_{\mathrm{s}}+\left(k_{\mathrm{b}}-k_{\mathrm{s}}\right) \mathbb{1}(t \geq \tau) & \text { if } K_{0}=k_{\mathrm{s}} \\
k_{\mathrm{b}} & \text { otherwise. }\end{cases}
\end{aligned}
$$

$W_{t}$ is the manager's continuation utility at time $t$. Following Sannikov (2008), this continuation utility is a natural state variable for the dynamic contracting problem that we consider below.

We call a contract $\Pi$ incentive-compatible and zero-savings if the solutions $\left\{\tilde{c}_{t}\right\}$ and $\left\{\tilde{i_{t}}\right\}$ to the manager's problem (A.1) are equal to the payment rule and the recommended incremental investment plan given in the contract. As is standard in the literature, without loss of generality, 
we focus on contracts in which the solution to problem (A.1) is to follow the recommended action level and maintain zero savings by virtue of the following version of the revelation principle.

Lemma A1. For an arbitrary contract $\tilde{\Pi}$, there is an incentive-compatible and zero-savings contract $\Pi$ that delivers at least as much value to the investor.

Next, we derive the necessary and sufficient conditions for a contract to be incentive-compatible and zero-savings. Following He (2011), we use the following intuition to first characterize the zerosavings condition. Suppose that $(\check{c}, \check{i})$ solves problem (A.1) for a given contract that implements zero savings. Further suppose that we simply endow the manager with savings $\mathcal{S}>0$ at some time $t>0$. How would her consumption and incremental investment plans respond? Because of the absence of wealth effects implied by the manager's CARA preferences, the optimal consumption plan for $s \geq t$ would be $\check{c}_{s}+r \mathcal{S}$, and the incremental investment plan would remain unchanged. Thus, an increase in savings from zero to $\mathcal{S}$ increases the manager's utility flow by a factor of $e^{-\gamma r S}$ forever. ${ }^{10}$ Put differently, the manager's marginal utility for an additional unit of savings when she currently has none is $-\gamma r$ multiplied by her current continuation utility. Moreover, for the manager to have no incentive to save, her marginal utility of consumption $u^{\prime}\left(\tilde{c}_{t}\right)$ must be equal to her marginal utility of savings $-\gamma r W_{t}$. This implies the following lemma:

Lemma A2. A contract is zero-savings if and only if

$$
u\left(\tilde{c}_{t}\right)=r W_{t} .
$$

For a given compensation $c_{t}$, recommended rate $i$, and actual rate $\tilde{i}_{t}$, the zero-savings condition implies that the manager's consumption is $\tilde{c}_{t}=c_{t}+\theta\left(g\left(i_{t}\right)-g\left(\tilde{i}_{t}\right)\right) X_{t} K_{t}$.

We now consider the incentive-compatibility condition. Standard martingale representation arguments suggest that the investor provides incentives by making the manager's continuation utility $W_{t}$ contingent on unexpected performance (see, e.g., Sannikov (2008)). For an arbitrary incentive-compatible and zero-savings contract, consider the following process:

$$
M_{t}=E_{t}\left[\int_{0}^{\infty} e^{-r s} u\left(c_{s}\right) d s\right] .
$$

\footnotetext{
${ }^{10}$ As utility is always negative, the factor $e^{-\gamma r S}<1$ represents an increase in utility.
} 
This process is clearly a martingale with respect to the filtration of public information $\mathcal{F}_{t}$; thus, the martingale representation theorem implies that progressively measurable processes $\beta_{t}$ exists such that the following holds:

$$
d M_{t}=-\gamma r W_{t} e^{-r t} \beta_{t}\left(d X_{t}-i_{t} X_{t} d t\right) .
$$

$M_{t}$ is related to the manager's continuation utility $W_{t}$ (under the recommended consumption and investment plans) as follows:

$$
d W_{t}=\left(r W_{t}-u\left(c_{t}\right)\right) d t+e^{r t} d M_{t}
$$

Combining the zero-savings condition (A.2) with Equations (A.3) and (A.4) gives the following dynamics for the manager's continuation utility:

$$
d W_{t}=-\gamma r W_{t} \beta_{t}\left(d X_{t}-i_{t} X_{t} d t\right)
$$

As a deviation from the recommended policy results in an unexpected (from the investor's perspective) shock to the growth of incremental capital, $\beta_{t}$ measures the manager's incentives to deviate from the contract's recommended policy.

For a given contract, problem (A.1) implies that the manager chooses the current rate of investment in incremental capital to maximize the sum of her instantaneous utility $u\left(c_{t}\right) d t$ and the expected change in her continuation utility $W_{t}{ }^{11}$ The manager's expected change in continuation utility from deviating from the recommended policy $i_{t}$ to $\tilde{i}_{t}$ is expressed as follows:

$$
E\left[d W_{t} \mid \tilde{i}\right]=\beta_{t}\left(-\gamma r W_{t}\right)\left(\tilde{i}-i_{t}\right) X_{t} d t .
$$

Thus, incentive compatibility requires the following:

$$
i_{t}=\arg \max _{\tilde{i}}\left\{u\left(\tilde{c}_{t}\right)+\beta_{t}\left(-\gamma r W_{t}\right)\left(\tilde{i}-i_{t}\right) X_{t}\right\}
$$

where $\tilde{c}_{t}=c_{t}+\theta\left(g\left(i_{t}\right)-g\left(\tilde{i}_{t}\right)\right) X_{t} K_{t}$. Taking a first-order derivative of the objective function in

\footnotetext{
${ }^{11}$ This argument is only heuristic. We provide a formal verification argument in the proof of Proposition A3.
} 
problem (A.6) with respect to $\tilde{i}$ and evaluating it at $\tilde{i}=i$ yields the following:

$$
\frac{\partial}{\partial \tilde{i}} u\left(c_{t}\right)+\beta_{t}\left(-\gamma r W_{t}\right) X_{t}
$$

As $\frac{\partial}{\partial i} u\left(c_{t}\right)=-u^{\prime}\left(c_{t}\right) \theta g^{\prime}\left(i_{t}\right) X_{t} K_{t}$ and the zero-savings condition is $u^{\prime}\left(c_{t}\right)=-\gamma r W_{t}$, we can simplify the first-order derivative above and find that it is constant in $i$ :

$$
\gamma r W_{t} \theta g^{\prime}\left(i_{t}\right) X_{t} K_{t}+\beta_{t}\left(-\gamma r W_{t}\right) X_{t}
$$

If this expression is strictly negative (positive), then only a minimal (maximal) investment rate is incentive-compatible. If this expression is zero, the manager is indifferent between all levels of $i$ in $\left[0, i_{\max }\right]$. We follow the usual convention that indifferent managers choose the recommended incremental investment rate. Thus, we obtain the following condition on incentive-compatible $\beta_{t}$ :

$$
\beta_{t} \begin{cases}\leq \theta g^{\prime}\left(i_{t}\right) K_{t} & \text { if } i_{t}=0 \\ =\theta g^{\prime}\left(i_{t}\right) K_{t} & \text { if } i_{t} \in\left(0, i_{\max }\right) \\ \geq \theta g^{\prime}\left(i_{t}\right) K_{t} & \text { if } i_{t}=i_{\max } .\end{cases}
$$

Intuitively, incentive compatibility requires the sensitivity of the manager's continuation utility to unexpected output shocks to be greater than or equal to her marginal cost of incremental investment $\theta g^{\prime}\left(i_{t}\right) X_{t} K_{t}$ scaled by the marginal effect of incremental investment on output $X_{t}$. Lemma A3 characterizes an incentive-compatible zero-savings contract.

Lemma A3. A contract is incentive-compatible and zero-savings if and only if the solution $W_{t}$ to problem (A.1) has the dynamics given by (A.5), where $\beta_{t}$ satisfies (A.7).

It is useful to represent the manager's continuation utility $W_{t}$ in terms of its certainty equivalent, $Y_{t}=-1 /(\gamma r) \ln \left(-\gamma r W_{t}\right)$. Note that we can use $Y_{t}$ as a state variable for the investor's problem in place of $W_{t}$, as $Y_{t}$ is a deterministic function of $W_{t}$. Applying Ito's lemma to (A.5) and combining it with Lemma A3 demonstrates that the dynamics of $Y_{t}$ under an incentive-compatible zero-savings contract are given by the following:

$$
d Y_{t}=\frac{1}{2} \gamma r \sigma^{2} \beta_{t}^{2} X_{t}^{2} d t+\sigma \beta_{t} X_{t} d Z_{t}
$$


The drift term in Equation (A.8) comes from the difference in risk aversion between the investor and the manager. As the manager is risk averse, the certainty equivalent of $W$ must have additional drift for each additional unit of volatility. As $W$ is a martingale, the drift term in $Y$ is entirely due to this effect. This positive drift shows up in the investor's Hamilton-Jacobi-Bellman (HJB) equation as the cost of providing incentives. We also impose an integrability restriction on $\beta_{t}$, which is detailed in the proof of Proposition 4 in Appendix B.

Next, we characterize the payment rule to the manager. Recall that the zero-savings condition in equation (A.2) provides a link between instantaneous utility and continuation utility. This allows us to express the manager's compensation as a function of the current state of the certainty equivalent of her continuation utility $Y_{t}$ as follows:

$$
c_{t}=r Y_{t}
$$

The first term in equation (A.9) is the cost of investing in incremental capital and the second is the risk-free rate times the certainty equivalent of her continuation utility. In other words, the contract pays the manager investment expenses at the recommended level plus the yield on her continuation utility. Given Equations (A.8) and (A.9), we can describe any incentive-compatible zero-savings contract by $(\beta, i, \tau)$.

We now move to the investor's problem. Denote by $v(x, y, k)$ the investor's value that solves objective (17). The problem can be simplified by noting that due to the absence of wealth effects implied by the CARA preferences of the manager, maximizing the investor's payoff is equivalent to maximizing the value function of the investor plus the certainty equivalent of the manager's continuation utility. Thus, rather than dealing directly with the investor's value function, we maximize the total firm value $V(x, k)$ :

$$
V(x, k)=v(x, y, k)+y
$$

The dependence on $y$ cancels out, as the risk-neutral investor values the manager's consumption stream at exactly its certainty equivalence. An application of Ito's formula then yields that over any interval of time in which there is no investment in physical capital, $V(x, k)$ must satisfy the 
HJB equation, Equation (19). We also observe that due to the concavity of the value function, the investor would never expose the manager to more risk than is required to provide incentives. Thus, the optimal contract would always set $\beta_{t}=0$ for $i_{t}=0$, and $\beta_{t}=\theta g^{\prime}\left(i_{t}\right) K_{t}$ otherwise.

\section{A.2 Solutions for Value Functions in Section 4.2}

In this appendix, we solve the value function and optimal contract for the model given in Section 4.2. The derivation follows the standard methods for solving real option exercise problems (see Dixit and Pindyck (1994) for an introduction to the topic). For ease of exposition, we assume that $\theta<\frac{1}{r}$; the case of $\theta>\frac{1}{r}$ is similar.

First, consider the optimal contract after the real option has been exercised. We hypothesize that there exists a threshold $x_{\mathrm{b}}^{*}$ such that the optimal incremental capital investment rate is given by Equation (21). The threshold $x_{\mathrm{b}}^{*}$ and firm value after the exercise of the real option are given by the solution to the following equations:

$$
\begin{aligned}
r V\left(x, k_{\mathrm{b}}\right)= & x k_{\mathrm{b}}\left(1-\theta i_{\max }\right)-\frac{1}{2} \gamma r\left(\theta \sigma x k_{\mathrm{b}}\right)^{2}+i_{\max } x V_{x}\left(x, k_{\mathrm{b}}\right) \\
& +\frac{1}{2} \sigma^{2} x^{2} V_{x x}\left(x, k_{\mathrm{b}}\right) \text { for } x<x_{\mathrm{b}}^{*} \\
r V\left(x, k_{\mathrm{b}}\right)= & x k_{\mathrm{b}}+\frac{1}{2} \sigma^{2} x^{2} V_{x x}\left(x, k_{\mathrm{b}}\right) \text { for } x \geq x_{\mathrm{b}}^{*} \\
\lim _{x \rightarrow x_{\mathrm{b}}^{*-}} V\left(x, k_{\mathrm{b}}\right)= & \lim _{x \rightarrow x_{\mathrm{b}}^{*+}} V\left(x, k_{\mathrm{b}}\right) \\
\lim _{x \rightarrow x_{\mathrm{b}}^{*-}} x V_{x}\left(x, k_{\mathrm{b}}\right)= & \lim _{x \rightarrow x_{\mathrm{b}}^{*+}} x V_{x}\left(x, k_{\mathrm{b}}\right) \\
\lim _{x \rightarrow x_{\mathrm{b}}^{*-}} x^{2} V_{x x}\left(x, k_{\mathrm{b}}\right)= & \lim _{x \rightarrow x_{\mathrm{b}}^{*+}} x^{2} V_{x x}\left(x, k_{\mathrm{b}}\right)
\end{aligned}
$$

Equations (A.12) and (A.13) are the value-matching and smooth-pasting conditions, respectively. Equation (A.14) is a hyper-contact condition that guarantees the optimality of $x_{\mathrm{b}}^{*}$. Equations (A.14)-(A.14) imply that the hypothesized control satisfies the HJB equation given in Equation (19) and must therefore be optimal. 
The solution to Equations (A.10)-(A.14) is given by

$$
V\left(x, k_{b}\right)=\left\{\begin{array}{l}
\left(\frac{\varepsilon+1}{\eta+\varepsilon}\right) h_{1}(x) B x k_{\mathrm{b}}-\left(\frac{\varepsilon+2}{\eta+\varepsilon}\right) h_{2}(x) A x^{2} k_{\mathrm{b}}^{2} \text { for } x \leq x_{\mathrm{b}}^{*} \\
\left(\left(\frac{\varepsilon+1}{\eta+\varepsilon}\right) \frac{1}{r B}+\left(\frac{\eta-1}{\eta+\varepsilon}\right) d_{1}(x)\right) B x k_{\mathrm{b}}-\left(\frac{\eta-2}{\eta+\varepsilon}\right) d_{2}(x) A x^{2} k_{\mathrm{b}}^{2} \text { for } x>x_{\mathrm{b}}^{*}
\end{array}\right.
$$

where

$$
\begin{aligned}
A & =\frac{\gamma r(\theta \sigma)^{2}}{2\left(r-2 i_{\max }-\sigma^{2}\right)}, \\
B & =\frac{1-\theta i_{\max }}{r-i_{\max }}, \\
h_{1}(x) & =\left(\frac{\eta+\varepsilon}{\varepsilon+1}\right)+\left(\frac{1}{r B}-1\right)\left(\frac{x}{x_{\mathrm{b}}^{*}}\right)^{\eta-1}, \\
h_{2}(x) & =\left(\frac{\eta+\varepsilon}{\varepsilon+2}\right)-\left(\frac{x}{x^{*}}\right)^{\eta-2}, \\
d_{1}(x) & =\left(\frac{1}{r B}\right)+\left(1-\frac{1}{r B}\right)\left(\frac{x}{x_{\mathrm{b}}^{*}}\right)^{-(\varepsilon+1)}, \\
d_{2}(x) & =\left(\frac{x}{x_{\mathrm{b}}^{*}}\right)^{-(\varepsilon+2)}, \\
x_{\mathrm{b}}^{*} & =\left(\frac{\eta-1}{\eta-2}\right)\left(\frac{\varepsilon+1}{\varepsilon+2}\right)\left(B-\frac{1}{r}\right)\left(\frac{1}{A k_{\mathrm{b}}}\right), \\
\varepsilon & =-\frac{1}{2}+\sqrt{\frac{1}{4}+\frac{2 r}{\sigma^{2}}},
\end{aligned}
$$

and

$$
\eta=-\left(\frac{i_{\max }}{\sigma^{2}}-\frac{1}{2}\right)+\sqrt{\left(\frac{i_{\max }}{\sigma^{2}}-\frac{1}{2}\right)^{2}+\frac{2 r}{\sigma^{2}}} .
$$

Note that $x_{\mathrm{b}}^{*}$ solves the following first-order condition

$$
(\eta-1) B \frac{\partial d_{1}(x)}{\partial x_{\mathrm{b}}^{*}} x k_{\mathrm{b}}-(\eta-2) A \frac{\partial d_{2}(x)}{\partial x_{\mathrm{b}}^{*}} x^{2} k_{\mathrm{b}}^{2}=0
$$

Next, we characterize the value function for the firm before investment, along with the optimal investment threshold $\bar{x}$. As discussed in Section 4.2, we restrict our attention to the parameters that imply that the optimal contract recommends a full rate of investment in incremental capital for all $x \leq \bar{x}$. 
Assumption A1. The price of new capital is not too high relative to the severity of the moral hazard problem:

$$
p \leq \frac{L}{\gamma}
$$

where $L$ is the positive constant given in Equation (B.45) of Appendix B. The expansion option is not too small.

$$
\frac{k_{\mathrm{b}}}{k_{\mathrm{s}}}>r B
$$

The above assumption directly implies that the optimal contract calls for maximal investment in incremental capital before expansion, as stated in the following Lemma.

Lemma A4. If Assumption A1 holds, then $i^{*}\left(x, k_{\mathrm{s}}\right)=i_{\max }$ for all $x \leq \bar{x}$.

Two important aspects of Assumption A1 require more explanation. First, the constant $L$ is such that the assumption is not overly restrictive. Indeed, all of the numerical examples that we consider below parameterize the model so that it satisfies the assumption. Second, the assumption is not necessary to guarantee that $i^{*}\left(x, k_{\mathrm{s}}\right)=i_{\max }$ for all $x \leq \bar{x}$. Restricting the parameters to the case in which the optimal contract calls for $i=i_{\max }$ for all $x \leq \bar{x}$, the value function is given by the following:

$$
\begin{aligned}
r V\left(x, k_{\mathrm{s}}\right) & =x k_{\mathrm{s}}(1-\theta)-\frac{1}{2} \gamma r\left(\theta \sigma x k_{\mathrm{s}}\right)^{2}+i_{\max } x V_{x}\left(x, k_{\mathrm{s}}\right)+\frac{1}{2} \sigma^{2} x^{2} V_{x x}\left(x, k_{\mathrm{s}}\right), \\
V\left(\bar{x}, k_{\mathrm{s}}\right) & =V\left(\bar{x}, k_{\mathrm{b}}\right)-p\left(k_{\mathrm{b}}-k_{\mathrm{s}}\right) \\
V_{x}\left(\bar{x}, k_{\mathrm{s}}\right) & =V_{x}\left(\bar{x}, k_{\mathrm{b}}\right) .
\end{aligned}
$$

The solution to Equations (A.27)-(A.29) is of the form

$$
V\left(x, k_{\mathrm{s}}\right)=B x k_{\mathrm{s}}-A x^{2} k_{\mathrm{s}}^{2}+\mathcal{C}_{\mathrm{s}} x^{\eta}
$$

where $\mathcal{C}_{\mathrm{s}}$ is a constant and $A$ and $B$ are as defined in Equations (A.16) and (A.15). Moreover, if there are multiple solutions to the boundary conditions given in Equations (A.28) and (A.29), then each solution yields a candidate value function. These candidates differ only in the constant coefficient $\mathcal{C}_{\mathrm{s}}$. Thus, the optimal value function is given by the solution with the largest such constant. 
We can combine conditions (A.28) and (A.29) to obtain

$$
\begin{aligned}
&(\eta-1) B\left(k_{\mathrm{b}}-k_{\mathrm{s}}\right) \bar{x}-(\eta-2) A\left(k_{\mathrm{b}}^{2}-k_{\mathrm{s}}^{2}\right) \bar{x}^{2}=\eta p\left(k_{\mathrm{b}}-k_{\mathrm{s}}\right), \text { if } i^{*}\left(\bar{x}, k_{\mathrm{b}}\right)=i_{\max } \\
&(\eta-1) B\left(d_{1}(\bar{x}) k_{\mathrm{b}}-k_{\mathrm{s}}\right) \bar{x}-(\eta-2) A\left(d_{2}(\bar{x}) k_{\mathrm{b}}^{2}-k_{\mathrm{s}}^{2}\right) \bar{x}^{2}=\eta p\left(k_{\mathrm{b}}-k_{\mathrm{s}}\right), \text { if } i^{*}\left(\bar{x}, k_{\mathrm{b}}\right)=0
\end{aligned}
$$

The final step to determining the solution to Equations (A.27)-(A.29) is to determine $i^{*}\left(\bar{x}, k_{\mathrm{b}}\right)$. If $i^{*}\left(\bar{x}, k_{\mathrm{b}}\right)=i_{\max }$, Equation (A.30) either has two positive roots or no real roots. If there are no real roots, then $i^{*}\left(\bar{x}, k_{\mathrm{b}}\right)=i_{\max }$ is not optimal. By solving Equation (A.28) for $\mathcal{C}_{\mathrm{s}}$, we obtain the following:

$$
\left.\mathcal{C}_{\mathrm{s}}=\mathcal{C}_{\mathrm{s} 2}=\left(B\left(\left(\frac{\varepsilon+1}{\eta+\varepsilon}\right) h_{1}(\bar{x}) k_{\mathrm{b}}-k_{\mathrm{s}}\right) \bar{x}-A\left(\left(\frac{\varepsilon+2}{\eta+\varepsilon}\right) h_{2}(\bar{x}) k_{\mathrm{b}}^{2}-k_{\mathrm{s}}^{2}\right) \bar{x}^{2}-p\left(k_{\mathrm{b}}-k_{\mathrm{s}}\right)\right) \bar{x}^{-\eta}\right) .
$$

Taking the derivative of $\mathcal{C}_{\mathrm{s} 2}$ with respect to $\bar{x}$ yields the following:

$$
\frac{\partial \mathcal{C}_{\mathrm{s} 2}}{\partial \bar{x}}=\left(-(\eta-1) B\left(k_{\mathrm{b}}-k_{\mathrm{s}}\right) \bar{x}+(\eta-2) A\left(k_{\mathrm{b}}^{2}-k_{\mathrm{s}}^{2}\right) \bar{x}^{2}+\eta p\left(k_{\mathrm{b}}-k_{\mathrm{s}}\right)\right) \bar{x}^{-(\eta+1)}
$$

If there are no real roots of Equation (A.30), then Equation (A.32) is positive for any $\bar{x} \geq 0$. Thus, the value function $V_{\mathrm{s}}$, which depends on $\bar{x}$ only via $\mathcal{C}_{\mathrm{s}}$, increases in $\bar{x}$, and it is optimal to postpone option exercise until $x$ reaches $x_{\mathrm{b}}^{*}$ when $i^{*}\left(\bar{x}, k_{\mathrm{b}}\right)=0$. It follows that $i^{*}\left(\bar{x}, k_{\mathrm{b}}\right)=1$ cannot be optimal. Now consider the case in which the roots of Equation (A.30) are positive. To find the optimal threshold from the two positive roots, note that Equation (A.32) is negative between the two roots, and so $\mathcal{C}_{\mathrm{s} 2}$ is decreasing between the two candidate thresholds. Thus, the smaller root of Equation (A.30) is the only possible candidate for an optimal threshold. This root is given by

$$
\bar{x}_{1}=\frac{(\eta-1) B-\sqrt{((\eta-1) B)^{2}-4 \eta(\eta-2) p A\left(k_{\mathrm{b}}+k_{\mathrm{s}}\right)}}{2(\eta-2) A\left(k_{\mathrm{b}}+k_{\mathrm{s}}\right)} .
$$

If $i^{*}\left(\bar{x}, k_{\mathrm{b}}\right)=0$, then Equation (A.31) either has two positive roots or no real roots. In the event that Equation (A.31) has two positive roots, the smaller root is always less than $x_{\mathrm{b}}^{*}$, and thus the larger root, which we denote by $\bar{x}_{2}$, is the only candidate solution. If $\bar{x}_{1}$ is real, then $\bar{x}_{2} \leq \bar{x}_{1}$ because the left-hand side of Equation (A.30) is always weakly smaller than the left-hand side of Equation (A.31). This implies that if $\bar{x}_{1} \leq x_{\mathrm{b}}^{*}$, then $\bar{x}_{2} \leq x_{\mathrm{b}}^{*}$ and thus $i^{*}\left(\bar{x}_{2}, k_{\mathrm{b}}\right)=1$, which implies 
that $\bar{x}_{2}$ is not optimal, and $\bar{x}_{1}$ must be the optimal threshold.

To summarize, if $\bar{x}_{1} \leq x_{\mathrm{b}}^{*}$, then $\bar{x}_{2}$ is not a solution to Equations (A.28) and (A.29), and $\bar{x}_{1}$ is the optimal investment threshold. If $\bar{x}_{1} \geq x_{\mathrm{b}}^{*}$, then $\bar{x}_{1}$ is not a solution to Equations (A.28) and (A.29), and $\bar{x}_{2}$ is the optimal investment threshold. To determine when $\bar{x}_{1} \leq x_{\mathrm{b}}^{*}$, note that when $\gamma$ is small (large), $x_{\mathrm{b}}^{*}$ is large (small) and $\bar{x}_{1}$ is small (large), and $\bar{x}_{1}\left(\bar{x}_{2}\right)$ will be the optimal threshold. We state this result in the following lemma.

Lemma A5. There exists $\gamma_{1}$ such that the optimal investment threshold is given by

$$
\bar{x}= \begin{cases}\bar{x}_{1} & \text { if } \gamma \leq \gamma_{1} \\ \bar{x}_{2} & \text { otherwise }\end{cases}
$$

Finally, we introduce some additional notation that will be helpful in the proofs of our main results. Let $f_{1}(x)$ and $f_{2}(x)$ be given by

$$
\begin{aligned}
& f_{1}(x)=(\eta-1) B\left(k_{\mathrm{b}}-k_{\mathrm{s}}\right) x-(\eta-2) A\left(k_{\mathrm{b}}^{2}-k_{\mathrm{s}}^{2}\right) x^{2}-\eta p\left(k_{\mathrm{b}}-k_{\mathrm{s}}\right) \\
& f_{2}(x)=(\eta-1) B\left(d_{1}(\bar{x}) k_{\mathrm{b}}-k_{\mathrm{s}}\right) \bar{x}-(\eta-2) A\left(d_{2}(\bar{x}) k_{\mathrm{b}}^{2}-k_{\mathrm{s}}^{2}\right) \bar{x}^{2}-\eta p\left(k_{\mathrm{b}}-k_{\mathrm{s}}\right) .
\end{aligned}
$$

Note that $f_{1}(x)$ is concave because $(\eta-2) A>0$ and using the definitions of $d_{1}(x), d_{2}(x)$, and $x_{\mathrm{b}}^{*}$, we have

$$
f_{2}^{\prime \prime}(x)=2(\eta-1) A k_{\mathrm{s}}^{2}+\varepsilon\left(\frac{\varepsilon+1}{\varepsilon+2}\right)(\eta-1)\left(B-\frac{1}{r}\right) x_{\mathrm{b}}^{*}\left(\frac{x}{x_{\mathrm{b}}^{*}}\right)^{-(\varepsilon+2)}>0
$$

and thus $f_{2}(x)$ is convex. Moreover, $f_{1}\left(\bar{x}_{1}\right)=f_{2}\left(\bar{x}_{2}\right)=0$.

\section{Appendix B: Proofs}

Appendix B is divided into two parts. Section B.1 contains the proofs of the results presented in the main text of the paper. Section B.2 contains the proofs of the supporting results used throughout the appendix. 


\section{B.1 Proofs of Main Results}

Proof of Proposition 1. By the verification argument we provide in the proof of Proposition 4, Equation (5) with boundary conditions (8)-(10) is sufficient for optimality.

Proof of Proposition 2. The proof is in the text preceding the statement of the propositions.

Proof of Proposition 3. The case of $\theta<\frac{1}{r}$ is covered in the text. In the case of $\frac{1}{r}<\theta<\left(\frac{k_{\mathrm{b}}}{k_{\mathrm{s}}}\right) \frac{1}{r}$, first note that

$$
V\left(x, k_{\mathrm{b}}\right)=\frac{x k_{\mathrm{b}}}{r}
$$

Thus, the smooth-pasting condition in Equation (9) for $\bar{x}$ implies that

$$
\frac{\partial}{\partial i}\left(-\theta i \bar{x} k_{\mathrm{s}}+i \bar{x} V_{x}\left(\bar{x}, k_{\mathrm{s}}\right)\right)=\bar{x}\left(-\theta k_{\mathrm{s}}+\frac{k_{\mathrm{b}}}{r}\right)>0
$$

and Equation (7) implies that $i^{*}\left(\bar{x}, k_{\mathrm{s}}\right)=1$. Differentiating the smooth-pasting condition with respect to $\theta$ yields the following:

$$
\frac{\partial \bar{x}}{\partial \theta}\left(V_{x x}\left(\bar{x}, k_{\mathrm{b}}\right)-V_{x x}\left(\bar{x}, k_{\mathrm{s}}\right)\right)=V_{x \theta}\left(\bar{x}, k_{\mathrm{s}}\right)
$$

$V_{x x}\left(x, k_{\mathrm{b}}\right)=0$, and by the ODE in Equation (5), we obtain the following:

$$
\begin{aligned}
V_{x x}\left(\bar{x}, k_{\mathrm{s}}\right) & =\frac{r V\left(\bar{x}, k_{\mathrm{s}}\right)-\bar{x} k_{\mathrm{s}}\left(1-\theta i_{\max }\right)-i_{\max } \bar{x} V_{x}(\bar{x})}{\frac{1}{2} \sigma^{2} \bar{x}^{2}}, \\
& =\frac{\bar{x} k_{\mathrm{b}}\left(1-\frac{1}{r} i_{\max }\right)-\bar{x} k_{\mathrm{s}}\left(1-\theta i_{\max }\right)}{\frac{1}{2} \sigma^{2} \bar{x}^{2}}, \\
& >\frac{\bar{x} k_{\mathrm{b}}\left(1-\theta i_{\max }\right)-\bar{x} k_{\mathrm{s}}\left(1-\theta i_{\max }\right)}{\frac{1}{2} \sigma^{2} \bar{x}^{2}}>0
\end{aligned}
$$

such that we can rearrange Equation (B.1) and apply the one-sided version of l'Hôpital's rule to obtain the following:

$$
\frac{\partial \bar{x}}{\partial \theta}=\frac{V_{x \theta}\left(\bar{x}, k_{\mathrm{s}}\right)}{V_{x x}\left(\bar{x}, k_{\mathrm{b}}\right)-V_{x x}\left(\bar{x}, k_{\mathrm{s}}\right)}=\lim _{x \uparrow \bar{x}} \frac{V_{x \theta}\left(\bar{x}, k_{\mathrm{s}}\right)}{V_{x x}\left(\bar{x}, k_{\mathrm{b}}\right)-V_{x x}\left(\bar{x}, k_{\mathrm{s}}\right)}=\lim _{x \uparrow \bar{x}} \frac{V_{\theta}\left(\bar{x}, k_{\mathrm{s}}\right)}{V_{x}\left(\bar{x}, k_{\mathrm{b}}\right)-V_{x}\left(\bar{x}, k_{\mathrm{s}}\right)} .
$$

By the ODE given in Equation $(5), V_{\theta}\left(x, k_{s}\right)<0$. Moreover, we claim that there exists $\varepsilon>0$ such that $V_{x}\left(x, k_{\mathrm{b}}\right)-V_{x}\left(x, k_{\mathrm{s}}\right)>0$ for all $x \in(\bar{x}-\varepsilon, \bar{x})$. This claim implies that there exists $\varepsilon>0$ such 
that

$$
\frac{V_{\theta}\left(x, k_{\mathrm{s}}\right)}{V_{x}\left(x, k_{\mathrm{b}}\right)-V_{x}\left(x, k_{\mathrm{s}}\right)}<0
$$

for all $x \in(\bar{x}-\varepsilon, \bar{x})$, which in turn implies the following:

$$
\lim _{x \uparrow \bar{x}} \frac{V_{\theta}\left(x, k_{\mathrm{s}}\right)}{V_{x}\left(x, k_{\mathrm{b}}\right)-V_{x}\left(x, k_{\mathrm{s}}\right)} \leq 0,
$$

as $V_{\theta}\left(x, k_{\mathrm{s}}\right)$ and $V_{x}\left(x, k_{\mathrm{b}}\right)-V_{x}\left(x, k_{\mathrm{s}}\right)$ are nonzero and continuous. Thus, $\frac{\partial \bar{x}}{\partial \theta} \leq 0$.

We now prove the claim that there exists $\varepsilon>0$ such that $V_{x}\left(x, k_{\mathrm{b}}\right)-V_{x}\left(x, k_{\mathrm{s}}\right)>0$ for all $x \in$ $(\bar{x}-\varepsilon, \bar{x})$ by contradiction. Suppose that there does not exist $\varepsilon>0$ such that $V_{x}\left(x, k_{\mathrm{s}}\right)<V_{x}\left(x, k_{\mathrm{b}}\right)$ for all $x \in(\bar{x}-\varepsilon, \bar{x})$; then, for all $\varepsilon>0$, there exists $x \in(\bar{x}-\varepsilon, \bar{x})$ such that $V_{x}\left(x, k_{\mathrm{s}}\right) \geq V_{x}\left(x, k_{\mathrm{b}}\right)$. As $V_{x}\left(\bar{x}, k_{\mathrm{s}}\right) \geq V_{x}\left(\bar{x}, k_{\mathrm{b}}\right), V_{x}\left(x, k_{\mathrm{b}}\right)$, and $V_{x}\left(x, k_{\mathrm{b}}\right)$ are continuous, this implies that there exists $\varepsilon>0$ such that $V_{x}\left(x, k_{\mathrm{s}}\right) \geq V_{x}\left(x, k_{\mathrm{b}}\right)$ for all $x \in(\bar{x}-\varepsilon, \bar{x})$. This implies that for $x \in(\bar{x}-\varepsilon, \bar{x})$, we have the following:

$$
V\left(x, k_{\mathrm{b}}\right)-V\left(x, k_{\mathrm{s}}\right)=V\left(\bar{x}, k_{\mathrm{b}}\right)-V\left(\bar{x}, k_{\mathrm{s}}\right)-\int_{x}^{\bar{x}}\left(V_{x}\left(z, k_{\mathrm{b}}\right)-V_{x}\left(z, k_{\mathrm{s}}\right)\right) d z \geq p\left(k_{\mathrm{b}}-k_{\mathrm{s}}\right),
$$

which contradicts the definition of $\bar{x}$.

Proof of Propositions 1 and 4 . We first prove Proposition 4. The argument for Propisition 1 is a special case. The proof proceeds in three steps. In Step 1, we show that we can replace the investor's maximization problem (problem (B.7)) with one in which we maximize a function independent of $Y_{t}$ (problem (B.9)). In Step 2, we fix an exercise threshold and verify that the solution to the HJB equation solves problem (B.9) for this investment policy. In Step 3, we show that the optimal investment policy must be a threshold rule that satisfies the boundary conditions given in Equations (8)-(10). Finally, we have already verified that the proposed contract is incentive-compatible and zero-savings in the proof of Lemma A3. Before we complete these steps, we make the following technical assumption on $\beta_{t}$ :

$$
E\left[\int_{0}^{\infty} \beta_{t}^{2} X_{t}^{2} d t\right]<\infty
$$

where the expectation is computed with respect to the measure induced by the incentive-compatible dynamics of $X_{t}$ given $\beta_{t}$. This restriction rules out contracts under which the manager has incentives 
to exert maximal effort forever. However, such contracts would be infinitely costly to implement, so this is without loss of generality.

Step 1: Let $v(x, w, k)$ be the value to the investor under a given incentive-compatible zerosavings contract $(c, i, \tau)$ with $X_{0}=x$ and $W_{0}=w$. Formally, $v(x, w, k)$ solves

$$
v(x, w, k)=\max _{c, i, \tau} E\left[\int_{0}^{\infty} e^{-r t}\left(X_{t} K_{t}-\theta g\left(i_{t}\right) X_{t} K_{t}-c_{t}\right) d t-e^{-r \tau} P\right],
$$

such that $d X_{t}=\tilde{i}_{t} X_{t} d t+\sigma X_{t} d Z_{t}, \quad X_{0}=x$,

$$
\begin{array}{ll}
K_{t} & = \begin{cases}k_{\mathrm{s}}+\left(k_{\mathrm{b}}-k_{\mathrm{s}}\right) \mathbb{1}(t \geq \tau) & \text { if } k=k_{\mathrm{s}} \\
k_{\mathrm{b}} & \text { otherwise, }\end{cases} \\
w & \leq E\left[\int_{0}^{\infty}-\frac{1}{\gamma} e^{-\gamma \tilde{c}_{t}-r t} d t\right],
\end{array}
$$

where $(\tilde{c}, \tilde{a})$ solves problem (A.1). Lemmas A2 and A3 imply that the compensation process $c_{t}$ must be given by Equation (A.9). The investor's value is the present value of the cash flows of the firm net of compensation to the manager; thus, we have the following:

$$
\begin{aligned}
& v(x, w, k) \\
& =E\left[\int_{0}^{\infty} e^{-r t}\left(X_{t} K_{t}\left(1-\theta g\left(i_{t}\right)\right)-c_{t}\right) d t-e^{-r \tau} P \mid X_{0}=x, Y_{0}=-\frac{1}{\gamma r} \ln (-\gamma r w), K_{0}=k\right] \\
& =E\left[\int_{0}^{\infty} e^{-r t}\left(X_{t} K_{t}\left(1-\theta g\left(i_{t}\right)\right)-r Y_{t}\right) d t-e^{-r \tau} P\right] \\
& =E\left[\int_{0}^{\infty} e^{-r t} X_{t} K_{t}\left(1-\theta g\left(i_{t}\right)\right) d t-e^{-r \tau} P\right] \\
& +E\left[\int_{0}^{\infty} r e^{-r t}\left(Y_{0}+\int_{0}^{t} \frac{1}{2} \gamma r \sigma^{2} \beta_{s}^{2} X_{s}^{2} d s+\int_{0}^{t} \sigma \beta_{s} X_{s} d Z_{s}\right) d t\right],
\end{aligned}
$$

where the last line follows from the dynamics of $Y_{t}$ given in Equation (A.8) and the conditioning is suppressed for clarity. Separately evaluating the three terms of the last expectation above, we obtain the following:

$$
E\left[\int_{0}^{\infty} r e^{-r t} Y_{0} d t\right]=Y_{0}
$$




$$
\begin{aligned}
E\left[\int_{0}^{\infty} r e^{-r t} \int_{0}^{t} \frac{1}{2} \gamma r \sigma^{2} \beta_{s}^{2} X_{s}^{2} d s d t\right] & =E\left[\int_{0}^{\infty} \int_{s}^{\infty} r e^{-r t} \frac{1}{2} \gamma r \sigma^{2} \beta_{s}^{2} X_{s}^{2} d t d s\right] \\
& =E\left[\int_{0}^{\infty} e^{-r s} \frac{1}{2} \gamma r \sigma^{2} \beta_{s}^{2} X_{s}^{2} d s\right]
\end{aligned}
$$

and

$$
\begin{aligned}
E\left[\int_{0}^{\infty} r e^{-r t} \sigma \beta_{t} X_{t} d Z_{t} d t\right] & =\int_{0}^{\infty} r e^{-r t} E\left[\int_{0}^{t} \sigma \beta_{t} X_{t} d Z_{t}\right] d t \\
& =0 .
\end{aligned}
$$

We can exchange the order of integration in the second and third steps above by Fubini's theorem and the assumption given in Equation (B.6). Collecting the terms yields the following:

$$
v(x, w, k)=E\left[\int_{0}^{\infty} e^{-r t}\left(X_{t} K_{t}\left(1-\theta g\left(i_{t}\right)\right)-\frac{1}{2} \gamma r \sigma^{2} \beta_{t}^{2} X_{t}^{2}\right) d t-e^{-r \tau} P\right]-y,
$$

where $y=-\frac{1}{2} \gamma r \ln (-\gamma r w)$. Combining the above arguments with Lemma A3, problem (B.7) is equivalent to

$$
V(x, k)=\max _{\beta, \lambda, i, \tau} E\left[\int_{0}^{\infty} e^{-r t}\left(X_{t} K_{t}\left(1-\theta g\left(i_{t}\right)\right)-\frac{1}{2} \gamma r \sigma^{2} \beta_{t}^{2} X_{t}^{2}\right) d t-e^{-r \tau} P\right],
$$

such that $d X_{t}=i_{t} X_{t} d t+\sigma X_{t} d Z_{t}, \quad X_{0}=x$,

$$
K_{t}= \begin{cases}k_{\mathrm{s}}+\left(k_{\mathrm{b}}-k_{\mathrm{s}}\right) \mathbb{1}(t \geq \tau) & \text { if } k=k_{\mathrm{s}} \\ k_{\mathrm{b}} & \text { otherwise }\end{cases}
$$

and the incentive-compatibility condition, Equation (A.7). From problem (B.8), it is clear that it is optimal to set $\beta_{t}$ as low as possible in absolute value so that the incentive-compatibility constraints in (A.7) are binding for non-zero levels of $i$ and $\beta_{t}=0$ when $i$ equals 0 , as setting $\beta_{t}$ larger than necessary to provide incentives can only decrease the objective function. Thus, problem (B.8) further simplifies to the following:

$$
V(x, k)=\max _{a, \tau} E\left[\int_{0}^{\infty} e^{-r t}\left(X_{t} K_{t}\left(1-\theta g\left(i_{t}\right)\right)-\frac{1}{2} \gamma r \sigma^{2} \beta_{t}^{2} X_{t}^{2}\right) d t-e^{-r \tau} P\right],
$$


such that

$$
\begin{aligned}
d X_{t} & =\tilde{i}_{t} X_{t} d t+\sigma X_{t} d Z_{t}, \quad X_{0}=x \\
K_{t} & = \begin{cases}k_{\mathrm{s}}+\left(k_{\mathrm{b}}-k_{\mathrm{s}}\right) \mathbb{1}(t \geq \tau) & \text { if } k=k_{\mathrm{s}} \\
k_{\mathrm{b}} & \text { otherwise }\end{cases} \\
\beta_{t} & =\theta g^{\prime}\left(i_{t}\right) K_{t} \mathbb{1}\left(i_{t}>0\right) .
\end{aligned}
$$

Step 2: Fix an arbitrary investment rule $\hat{\tau}$ and let $\hat{V}$ and $\hat{i}$ solve the following:

$$
r \hat{V}=\max _{i} \mathcal{L}(x, k, \hat{V} ; i)
$$

where

$$
\mathcal{L}(x, k, V ; i)=x k(1-\theta g(i))-\frac{1}{2} \gamma r \sigma^{2} \beta^{2} x^{2}+i x \frac{\partial V}{\partial x}+\frac{1}{2} \sigma^{2} x^{2} \frac{\partial^{2} V}{\partial x^{2}},
$$

such that

$$
\begin{array}{r}
\beta=\theta g^{\prime}(i) k \mathbb{1}(i>0), \\
\hat{V}\left(X_{\tau}, k_{\mathrm{s}}\right) \stackrel{\text { a.s }}{=} \hat{V}\left(X_{\tau}, k_{\mathrm{b}}\right)-P .
\end{array}
$$

In words, $(\hat{i}, \hat{\tau})$ is the proposed optimal incentive-compatible zero-savings effort contract fixing the investment time $\hat{\tau}$ and $\hat{V}$ is the corresponding value function. Let $(\tilde{i}, \hat{\tau})$ be an arbitrary incentivecompatible zero-savings contract with the same investment time $\hat{\tau}$ and let

$$
G_{t}=\int_{0}^{t} e^{-r s}\left(\tilde{X}_{s} \tilde{K}_{s}\left(1-\theta g\left(\tilde{i}_{s}\right)\right)-\frac{1}{2} \gamma r \sigma^{2} \tilde{\beta}_{s}^{2} \tilde{X}_{s}^{2}\right) d s+e^{-r t} \hat{V}\left(\tilde{X}_{t}, \tilde{K}_{t}\right)-\mathbb{1}(t \leq \hat{\tau}) e^{-r \hat{\tau}} P
$$

where $G_{t}$ measures the gain in present value at time $t=0$ using the contract $(\tilde{i}, \hat{\tau})$ until time $t$ and then following the proposed optimal contract $(\hat{i}, \hat{\tau}) . \tilde{X}_{t}$ and $\tilde{K}_{t}$ are the productivity and capital, 
respectively, induced by the contract $(\tilde{i}, \hat{\tau})$. An application of Ito's lemma yields the following:

$$
e^{r t} d G_{t}=\left(\mathcal{L}\left(\tilde{X}_{t}, \tilde{K}_{t}, \hat{V} ; \tilde{i}_{t}\right)-r \hat{V}\right) d t+\sigma \tilde{X}_{t} \frac{\partial \hat{V}}{\partial x} d Z_{t}+\left(\hat{V}\left(X_{t}, k_{\mathrm{b}}\right)-\hat{V}\left(X_{t}, k_{\mathrm{s}}\right)-P\right) \mathbb{1}(t=\hat{\tau})
$$

The drift term given in (B.14) is always weakly negative by Equation (B.10) and the last term of (B.14) is always zero. Thus, $G_{t}$ is a supermartingale.

Consider the value from choosing the contract $(\tilde{i}, \hat{\tau})$. We have the following:

$$
\begin{aligned}
& E\left[\int_{0}^{\infty} e^{-r s}\left(\tilde{X}_{s} \tilde{K}_{s}\left(1-\theta g\left(\tilde{i}_{s}\right)\right)-\frac{1}{2} \gamma r \sigma^{2} \tilde{\beta}_{s}^{2} \tilde{X}_{s}^{2}\right) d s-e^{-r \hat{\tau}} P\right] \\
& =E\left[G_{t}\right]+e^{-r t} E\left[\int_{t}^{\infty} e^{-r(s-t)}\left(\tilde{X}_{s} \tilde{K}_{s}\left(1-\theta g\left(\tilde{i}_{s}\right)\right)-\frac{1}{2} \gamma r \sigma^{2} \tilde{\beta}_{s}^{2} \tilde{X}_{s}^{2}\right) d s-\hat{V}\left(\tilde{X}_{t}, \tilde{K}_{t}\right)\right] \\
& \leq G_{0}+e^{-r t} E\left[\int_{t}^{\infty} e^{-r(s-t)}\left(\tilde{X}_{s} \tilde{K}_{s}\left(1-\theta g\left(\tilde{i}_{s}\right)\right)-\frac{1}{2} \gamma r \sigma^{2} \tilde{\beta}_{s}^{2} \tilde{X}_{s}^{2}\right) d s-\hat{V}\left(\tilde{X}_{t}, \tilde{K}_{t}\right)\right] .
\end{aligned}
$$

The inequality follows from the fact that $G_{t}$ is a supermartingale. As $g\left(\tilde{i}_{s}\right) \geq 0$ and $\tilde{\beta}_{s}^{2} \tilde{X}_{s}^{2}>0$, we obtain the following:

$$
\begin{aligned}
E\left[\int_{t}^{\infty} e^{-r(s-t)}\left(\tilde{X}_{s} \tilde{K}_{s}\left(1-\theta g\left(\tilde{i}_{s}\right)\right)-\frac{1}{2} \gamma r \sigma^{2} \tilde{\beta}_{s}^{2} \tilde{X}_{s}^{2}\right) d s\right] & \leq E\left[\int_{t}^{\infty} e^{-r(s-t)} \tilde{X}_{s} \tilde{K}_{s} d s\right] \\
& \leq E\left[\int_{t}^{\infty} e^{-r(s-t)} \tilde{X}_{s} k_{\mathrm{b}} d s\right] \\
& \leq \frac{\tilde{X}_{t} k_{\mathrm{b}}}{r-i_{\max }}
\end{aligned}
$$

as the greatest possible expected present value of the gross (of effort and incentive cost) cash flow $\tilde{X}_{t} \tilde{K}$ is achieved when $\tilde{i}_{t}=i_{\max }$ and $K_{t}=k_{\mathrm{b}}$ for all $t$. Note that

$$
\hat{V}(x, k) \geq \frac{x k}{r}>0
$$


by Equation (B.10). Thus,

$$
\begin{aligned}
& E\left[\int_{0}^{\infty} e^{-r s}\left(\tilde{X}_{s} \tilde{K}_{s}\left(1-\theta g\left(\tilde{i}_{s}\right)\right)-\frac{1}{2} \gamma r \sigma^{2} \tilde{\beta}_{s}^{2} \tilde{X}_{s}^{2}\right) d s-e^{-r \hat{\tau}} P\right] \\
& \leq G_{0}+e^{-r t} E\left[\frac{\tilde{X}_{t} k_{\mathrm{b}}}{r-i_{\max }}\right] \\
& \leq G_{0}+e^{-\left(r-i_{\max }\right) t} \frac{X_{0} k_{\mathrm{b}}}{r-i_{\max }}
\end{aligned}
$$

where the last step again follows from the fact that the largest possible expected value for $\tilde{X}_{t}$ is achieved by setting $i_{s}=i_{\max }$ for all $s \leq t$ under which $\tilde{X}_{t}$ is a geometric Brownian motion so that $E\left[\tilde{X}_{t}\right]=X_{0} e^{i_{\max } t}$. Taking the limit as $t \rightarrow \infty$ of both sides yields the following:

$$
E\left[\int_{0}^{\infty} e^{-r s}\left(\tilde{X}_{s} \tilde{K}_{s}\left(1-\theta g\left(\tilde{i}_{s}\right)\right)-\frac{1}{2} \gamma r \sigma^{2} \tilde{\beta}_{s}^{2} \tilde{X}_{s}^{2}\right) d t-e^{-r \hat{\tau}} P\right] \leq G_{0}=\hat{V}\left(X_{0}, K_{0}\right)
$$

Thus, the contract $(\tilde{i}, \hat{\tau})$ yields a weakly lower value than the contract $(\hat{i}, \hat{\tau})$.

Next, we show that there is no loss of generality if we restrict our attention to solutions to Equations (B.10)-(B.12) with $i \in\left\{0, i_{\max }\right\}$ when $g(i)=i$. Suppose that $i \in\left(0, i_{\max }\right)$ solves Equation (B.10); then, $i$ must satisfy the following first-order condition:

$$
0=\frac{\partial \mathcal{L}(x, k, \hat{V} ; i)}{\partial i}=-\theta x k+x \frac{\partial \hat{V}}{\partial x}
$$

However, this implies that $\mathcal{L}(x, k, \hat{V} ; i)=\mathcal{L}\left(x, k, \hat{V} ; i^{\prime}\right)$ for all $i, i^{\prime}>0$, which implies that there is no loss of generality if we restrict our attention to the solution with $i \in\left\{0, i_{\max }\right\}$.

Step 3: Having established Steps 1 and 2, the resulting investment problem is a one-dimensional optimal stopping problem that satisfies standard Lipschitz and growth conditions. Thus, Theorem 4 of Strulovici and Szydlowski (2015) applies, and the value function is smooth and satisfies the boundary conditions given in Equations (8) and (9). The verification argument of Proposition 7 of Strulovici and Szydlowski (2015) applies, as the terminal payoff $V\left(x, k_{\mathrm{b}}\right)$ is twice continuously differentiable as established in Appendix A.2.

If we impose the constraint $\beta_{t}=0$ on problem (B.7), then we are left with problem (4). Nothing in the above argument relies on $\beta_{t} \neq 0$, so it also applies to problem (4), thus proving Proposition 1. 
Proof of Proposition 5. First consider $\gamma \leq \gamma_{1}$. Lemma A5 then implies $\bar{x}=\bar{x}_{1}$. Differentiating Equation (A.30) with respect to $\gamma$ and solving then gives

$$
\frac{\partial \bar{x}}{\partial \gamma}=-\frac{1}{f_{1}^{\prime}\left(\bar{x}_{1}\right)}\left(\frac{\partial f_{1}\left(\bar{x}_{1}\right)}{\partial \gamma}\right)
$$

Because $\bar{x}_{1}$ is the smaller root of the concave function $f_{1}(x)$, we have

$$
f_{1}^{\prime}\left(\bar{x}_{1}\right)>0
$$

Next, we have

$$
\frac{\partial f_{1}\left(\bar{x}_{1}\right)}{\partial \gamma}=-\frac{1}{\gamma}(\eta-2) A\left(k_{\mathrm{b}}^{2}-k_{\mathrm{s}}^{2}\right) \bar{x}_{1}^{2}<0
$$

Because $\left(r-2 i_{\max }-\sigma^{2}\right)(\eta-2)^{-1}=i_{\max }+\frac{1}{2}(\eta+1) \sigma^{2}>0$ by the definition $\eta$, so $(\eta-2) A>0$. Combining Equations (B.16) and (B.17) gives $\frac{\partial \bar{x}}{\partial \gamma}>0$.

Now consider $\gamma>\gamma_{1}$. Lemma A5 then implies that $\bar{x}=\bar{x}_{2}$. Differentiating Equation (A.31) with respect to $\gamma$ and solving then gives

$$
\frac{\partial \bar{x}}{\partial \gamma}=-\frac{1}{f_{2}^{\prime}\left(\bar{x}_{2}\right)}\left(\frac{\partial f_{2}\left(\bar{x}_{2}\right)}{\partial \gamma}\right)
$$

Because $\bar{x}_{2}$ is the larger root of the convex function $f_{2}(x)$, we have

$$
f_{2}^{\prime}\left(\bar{x}_{2}\right)>0 .
$$

Next, we have

$$
\begin{aligned}
\frac{\partial f_{2}\left(\bar{x}_{2}\right)}{\partial \gamma}= & \left((\eta-1) B \frac{\partial d_{1}\left(\bar{x}_{2}\right)}{\partial x_{\mathrm{b}}^{*}} \bar{x}_{2} k_{\mathrm{b}}-(\eta-2) A \frac{\partial d_{2}\left(\bar{x}_{2}\right)}{\partial x_{\mathrm{b}}^{*}} \bar{x}_{2}^{2} k_{\mathrm{b}}^{2}\right) \frac{\partial x_{\mathrm{b}}^{*}}{\partial \gamma} \\
& -\frac{1}{\gamma}(\eta-2) A\left(d_{2}\left(\bar{x}_{2}\right) k_{\mathrm{b}}^{2}-k_{\mathrm{s}}^{2}\right) \bar{x}_{2}^{2} .
\end{aligned}
$$


Substituting the optimality condition for $x_{\mathrm{b}}^{*}$ given in Equation (A.24) into (B.20) gives

$$
\frac{\partial f_{2}\left(\bar{x}_{2}\right)}{\partial \gamma}=-\frac{1}{\gamma}(\eta-2) A\left(d_{2}\left(\bar{x}_{2}\right) k_{\mathrm{b}}^{2}-k_{\mathrm{s}}^{2}\right) \bar{x}_{2}^{2}
$$

Because $(\eta-2) A>0$, the sign of $\frac{\partial f_{2}\left(\bar{x}_{2}\right)}{\partial \gamma}$ is determined by the sign of $d_{2}\left(\bar{x}_{2}\right) k_{\mathrm{b}}^{2}-k_{\mathrm{s}}^{2}$. For $\gamma=\gamma_{1}$, we have $\bar{x}_{2}=x_{\mathrm{b}}^{*}$, so

$$
\left[d_{2}\left(\bar{x}_{2}\right) k_{\mathrm{b}}^{2}-k_{\mathrm{s}}^{2}\right]_{\gamma=\gamma_{1}}=k_{\mathrm{b}}-k_{\mathrm{s}}>0 .
$$

Next, we have $\lim _{\gamma \rightarrow \infty} x_{\mathrm{b}}^{*}=0$ and $\lim _{\gamma \rightarrow \infty} \bar{x}_{2}>0$, so

$$
\lim _{\gamma \rightarrow \infty}\left(d_{2}\left(\bar{x}_{2}\right) k_{\mathrm{b}}^{2}-k_{\mathrm{s}}^{2}\right)=-k_{\mathrm{s}}
$$

Now, let $\gamma_{2}$ solve

$$
d_{2}\left(\left.\bar{x}_{2}\right|_{\gamma=\gamma_{2}}\right) k_{\mathrm{b}}^{2}-k_{\mathrm{s}}^{2}=0
$$

Rearranging Equation (B.24) gives that

$$
\left.\bar{x}_{2}\right|_{\gamma=\gamma_{2}}=\left.x_{\mathrm{b}}^{*}\right|_{\gamma=\gamma_{2}}\left(\frac{k_{\mathrm{s}}}{k_{\mathrm{b}}}\right)^{-\frac{2}{\varepsilon+2}} \text {. }
$$

Substituting Equations (B.24) and (B.25) into Equation (A.31) yields

$$
\left.x_{\mathrm{b}}^{*}\right|_{\gamma=\gamma_{2}}=\frac{p\left(k_{\mathrm{b}}-k_{\mathrm{s}}\right)\left(\frac{\eta}{\eta-1}\right)\left(\frac{k_{\mathrm{s}}}{k_{\mathrm{b}}}\right)^{\frac{2}{\varepsilon+2}}}{\left(\frac{1}{r}+\left(B-\frac{1}{r}\right)\left(\frac{k_{\mathrm{s}}}{k_{\mathrm{b}}}\right)^{\frac{\varepsilon+2}{\varepsilon+1}}\right) k_{\mathrm{b}}-B k_{\mathrm{s}}}
$$

which together with Equation (A.21) gives the following unique solution for $\gamma_{2}$

$$
\gamma_{2}=\left(\frac{\left(\frac{1}{r}+\left(B-\frac{1}{r}\right)\left(\frac{k_{\mathrm{s}}}{k_{\mathrm{b}}}\right)^{\frac{\varepsilon+2}{\varepsilon+1}}\right) k_{\mathrm{b}}-B k_{\mathrm{s}}}{p\left(k_{\mathrm{b}}-k_{\mathrm{s}}\right)\left(\frac{\eta}{\eta-1}\right)\left(\frac{k_{\mathrm{s}}}{k_{\mathrm{b}}}\right)^{\frac{2}{\varepsilon+2}}}\right)\left(\frac{\eta-1}{\eta-2}\right)\left(\frac{\varepsilon+1}{\varepsilon+2}\right)\left(B-\frac{1}{r}\right)\left(\frac{2\left(r-2 i_{\max }-\sigma^{2}\right)}{k_{\mathrm{b}}(\theta \sigma)^{2}}\right) .
$$

Note that $\gamma_{2}<\infty$ because $k_{\mathrm{b}}>k_{\mathrm{s}}$ and $\left(r-2 i_{\max }-\sigma^{2}\right)(\eta-2)^{-1}=i_{\max }+\frac{1}{2}(\eta+1) \sigma^{2}$ by the 
definition of $\eta$. Note that $\gamma_{2}>0$ if and only if

$$
\frac{1}{r}+\left(B-\frac{1}{r}\right)\left(\frac{k_{\mathrm{s}}}{k_{\mathrm{b}}}\right)^{\frac{\varepsilon+2}{\varepsilon+1}} k_{\mathrm{b}} \geq B\left(\frac{k_{\mathrm{s}}}{k_{\mathrm{b}}}\right)
$$

Because $d_{2}\left(\bar{x}_{2}\right)$ is continuous in $\gamma$, Equations (B.22) and (B.23) then imply that $d_{2}\left(\bar{x}_{2}\right) k_{\mathrm{b}}-k_{\mathrm{s}} \leq 0$ if and only if $\gamma \geq \gamma_{2}$. Moreover, note that $\left[d_{2}\left(\bar{x}_{2}\right) k_{\mathrm{b}}-k_{\mathrm{s}}\right]_{\gamma=\gamma_{1}}>0$, so $\gamma_{1}<\gamma_{2}$. As a result,

$$
\frac{\partial \bar{x}}{\partial \gamma} \begin{cases}\leq 0 & \text { if } \gamma<\gamma_{2} \\ >0 & \text { otherwise }\end{cases}
$$

which completes the proof.

Proof of Proposition 6. First note that $\left.\bar{x}\right|_{\gamma=0}=\bar{x}^{F B}$. Thus, if $\gamma_{2} \leq 0$, then $\bar{x}<\bar{x}^{F B}$ for all $\gamma$ by Proposition 5. In this case, $\gamma_{3}=0$.

If $\gamma_{2}>0$, then let $\gamma_{3}>0$ be a constant such that

$$
\left.f_{2}\left(\bar{x}^{F B}\right)\right|_{\gamma=\gamma_{3}}=0
$$

Note that $\left.f_{2}\left(\bar{x}^{F B}\right)\right|_{\gamma=0}=0, \bar{x}$ is increasing for $0<\gamma<\gamma_{2}$, and $f_{2}(x)$ is decreasing in $x$, so $\left.f_{2}\left(\bar{x}^{F B}\right)\right|_{\gamma=0}>\left.f_{2}(\bar{x})\right|_{\gamma=0}=0$ and $\gamma_{3}>\gamma_{2}$. Next note that $f_{2}(x)$ is decreasing in $\gamma$ for all $x$ and that $\lim _{\gamma \rightarrow \infty} f_{2}(x)=-\infty$, so there is a unique finite solution for $\gamma_{3}$ in Equation (B.30).

Proposition 5 implies that $\bar{x}$ is decreasing in $\gamma$ for all $\gamma \geq \gamma_{3}$ because $\gamma_{3}>\gamma_{2}$. Moreover, by the definition of $\gamma_{3}, x^{\mathrm{FB}}$ is the larger root of Equation (A.31), and thus $\bar{x}=\bar{x}^{\mathrm{FB}}$. Because $\bar{x}$ is decreasing for all $\gamma \geq \gamma_{3}$, it must be that $\bar{x}<\bar{x}^{\mathrm{FB}}$ for $\gamma>\gamma_{3}$.

Proof of Proposition 7. Note that $r>2 i_{\max }+\sigma^{2}$ implies $A>0$ and $\eta>2$. Let

$$
\gamma_{4}=\max \left\{\gamma_{2},\left(\frac{\sigma \eta p\left(k_{\mathrm{b}}-k_{\mathrm{s}}\right)}{i_{\max }+\frac{1}{2}(2 \eta-1) \sigma^{2}}\right)\left[\left(\frac{p\left(k_{\mathrm{b}}-k_{\mathrm{s}}\right) k_{\mathrm{s}}}{B k_{\mathrm{b}}}\right)\left(\frac{i_{\max } r(\theta \sigma)^{2}}{\left(i_{\max }+\frac{1}{2}(\eta+1) \sigma^{2}\right)^{2}}\right)\right]^{-1}\right\} .
$$

and let $\gamma \geq \gamma_{4}$. First note that $i^{*}\left(\bar{x}, k_{\mathrm{b}}\right)=0$, so $\bar{x}$ is given by Equation (A.31). Differentiating both 
sides of Equation (A.31) and solving for $\frac{\partial \bar{x}}{\partial \sigma}$ gives

$$
\frac{\partial \bar{x}}{\partial \sigma}=-\frac{1}{f_{2}^{\prime}\left(\bar{x}_{2}\right)}\left(\frac{\partial f_{2}\left(\bar{x}_{2}\right)}{\partial \sigma}\right)
$$

In the proof of Proposition 5, we show that $f_{2}^{\prime}\left(\bar{x}_{2}\right)>0$. We claim that $\gamma>\gamma_{4}$ implies $\frac{\partial f_{2}\left(\bar{x}_{2}\right)}{\partial \sigma}>0$ and thus also implies $\frac{\partial \bar{x}}{\partial \sigma}<0$. We have

$$
\begin{aligned}
\frac{\partial f_{2}\left(\bar{x}_{2}\right)}{\partial \sigma}= & \frac{\partial \eta}{\partial \sigma}\left(B\left(d_{1}(x) k_{\mathrm{b}}-k_{\mathrm{s}}\right) \bar{x}-A\left(d_{2}(\bar{x}) k_{\mathrm{b}}^{2}-k_{\mathrm{s}}^{2}\right) \bar{x}^{2}-P\right) \\
& -(\eta-2) \frac{\partial A}{\partial \sigma}\left(d_{2}(\bar{x}) k_{\mathrm{b}}^{2}-k_{\mathrm{s}}^{2}\right) \bar{x}^{2} \\
& +(\eta-1) B\left(\frac{\partial d_{1}(x)}{\partial \varepsilon} \frac{\partial \varepsilon}{\partial \sigma}+\frac{\partial d_{1}(x)}{\partial x_{\mathrm{b}}^{*}} \frac{\partial x_{\mathrm{b}}^{*}}{\partial \sigma}\right) x k_{b} \\
& -(\eta-2) A\left(\frac{\partial d_{2}(x)}{\partial \varepsilon} \frac{\partial \varepsilon}{\partial \sigma}+\frac{\partial d_{2}(x)}{\partial x_{\mathrm{b}}^{*}} \frac{\partial x_{\mathrm{b}}^{*}}{\partial \sigma}\right) x^{2} k_{b}^{2} . \\
\frac{\partial f_{2}\left(\bar{x}_{2}\right)}{\partial \sigma}= & \frac{\partial f_{2}\left(\bar{x}_{2}\right)}{\partial x_{\mathrm{b}}^{*}} \frac{\partial x_{\mathrm{b}}^{*}}{\partial \sigma}+\frac{\partial f_{2}\left(\bar{x}_{2}\right)}{\partial \varepsilon} \frac{\partial \varepsilon}{\partial \sigma}+\frac{\partial f_{2}\left(\bar{x}_{2}\right)}{\partial A} \frac{\partial A}{\partial \sigma}+\frac{\partial f_{2}\left(\bar{x}_{2}\right)}{\partial \eta} \frac{\partial \eta}{\partial \sigma}
\end{aligned}
$$

The optimality condition for $x_{\mathrm{b}}^{*}$ given in Equation (A.24) gives $\frac{\partial f_{2}\left(\bar{x}_{2}\right)}{\partial x_{\mathrm{b}}^{*}}=0$. Next, we have

$$
\begin{aligned}
\frac{\partial f_{2}\left(\bar{x}_{2}\right)}{\partial \varepsilon} & =\left((\eta-1) B \frac{\partial d_{1}(x)}{\partial \varepsilon} x k_{\mathrm{b}}-(\eta-2) A \frac{\partial d_{2}(x)}{\partial \varepsilon} x^{2} k_{\mathrm{b}}^{2}\right) \frac{\partial \varepsilon}{\partial \sigma} \\
& =-\left((\eta-1)\left(B-\frac{1}{r}\right)-(\eta-1) A x_{\mathrm{b}}^{*} k_{\mathrm{b}}\right)\left(\frac{\bar{x}}{x_{\mathrm{b}}^{*}}\right)^{-\varepsilon} \log \left(\frac{\bar{x}}{x^{*}}\right) x_{\mathrm{b}}^{*} k_{\mathrm{b}} \frac{\partial \varepsilon}{\partial \sigma} \\
& =-\left(\frac{\eta-1}{\varepsilon+2}\right)\left(B-\frac{1}{r}\right)\left(\frac{\bar{x}}{x_{\mathrm{b}}^{*}}\right)^{-\varepsilon} \log \left(\frac{\bar{x}}{x^{*}}\right) x_{\mathrm{b}}^{*} k_{\mathrm{b}} \frac{\partial \varepsilon}{\partial \sigma}
\end{aligned}
$$

where the last step follows from the definition of $x_{\mathrm{b}}^{*}$. Observe that $\varepsilon>0, \eta>1, B>\frac{1}{r}, \bar{x}>x_{\mathrm{b}}^{*}$, and $\frac{\partial \varepsilon}{\partial \sigma}<0$, so Equation (B.32) implies

$$
\frac{\partial f_{2}\left(\bar{x}_{2}\right)}{\partial \varepsilon} \frac{\partial \varepsilon}{\partial \sigma}>0
$$

We can use the definition of $A$ to obtain

$$
\frac{\partial f_{2}\left(\bar{x}_{2}\right)}{\partial A} \frac{\partial A}{\partial \sigma}=-(\eta-2)\left(\frac{2\left(r-2 i_{\max }\right)}{\sigma\left(r-2 i_{\max }-\sigma^{2}\right)}\right) A\left(d_{2}(\bar{x}) k_{\mathrm{b}}^{2}-k_{\mathrm{s}}^{2}\right) \bar{x}^{2}>0,
$$


because $\gamma>\gamma_{2}$ implies $d_{2}(\bar{x}) k_{\mathrm{b}}^{2}-k_{\mathrm{s}}^{2}<0$.

Next, we have

$$
\begin{aligned}
\frac{\partial f_{2}\left(\bar{x}_{2}\right)}{\partial \eta} & =B\left(d_{1}(\bar{x}) k_{\mathrm{b}}-k_{\mathrm{s}}\right) \bar{x}-A\left(d_{2}(\bar{x}) k_{\mathrm{b}}^{2}-k_{\mathrm{s}}^{2}\right) \bar{x}^{2}-p\left(k_{\mathrm{b}}-k_{\mathrm{s}}\right) \\
& =\left(\frac{1}{\eta-1}\right)\left(-A\left(d_{2}(\bar{x}) k_{\mathrm{b}}^{2}-k_{\mathrm{s}}^{2}\right) \bar{x}^{2}+p\left(k_{\mathrm{b}}-k_{\mathrm{s}}\right)\right)
\end{aligned}
$$

where the last step follows from applying Equation (A.31). We thus have

$$
\begin{aligned}
\frac{\partial f_{2}\left(\bar{x}_{2}\right)}{\partial A} \frac{\partial A}{\partial \sigma}+\frac{\partial f_{2}\left(\bar{x}_{2}\right)}{\partial \eta} \frac{\partial \eta}{\partial \sigma}= & -\left((\eta-2)\left(\frac{2\left(r-2 i_{\max }\right)}{\sigma\left(r-2 i_{\max }-\sigma^{2}\right)}\right)+\frac{1}{\eta-1} \frac{\partial \eta}{\partial \sigma}\right) A\left(d_{2}(\bar{x}) k_{\mathrm{b}}^{2}-k_{\mathrm{s}}^{2}\right) \bar{x}^{2} \\
& +\frac{p\left(k_{\mathrm{b}}-k_{\mathrm{s}}\right)}{\eta-1} \frac{\partial \eta}{\partial \sigma}
\end{aligned}
$$

We have

$$
\begin{aligned}
\frac{\partial \eta}{\partial \sigma} & =-\frac{\sigma \eta(\eta-1)}{i_{\max }+\frac{1}{2}(2 \eta-1) \sigma^{2}}, \\
r-2 i_{\max }-\sigma^{2} & \left.=(\eta-2)\left(i_{\max }+\frac{1}{2}(\eta+1) \sigma^{2}\right)\right), \\
r-2 i_{\max } & =(\eta-2) i_{\max }+\frac{1}{2} \eta(\eta-1) \sigma^{2},
\end{aligned}
$$

which together imply

$$
\begin{aligned}
(\eta-2)\left(\frac{2\left(r-2 i_{\max }\right)}{\sigma\left(r-2 i_{\max }-\sigma^{2}\right)}\right)+\frac{1}{\eta-1} \frac{\partial \eta}{\partial \sigma} & =\frac{2(\eta-2) \frac{i_{\max }}{\sigma}+\eta(\eta-1) \sigma}{i_{\max }+\frac{1}{2}(\eta+1) \sigma^{2}}-\frac{\eta \sigma}{i_{\max }+\frac{1}{2}(2 \eta-1) \sigma^{2}} \\
& \geq \frac{2(\eta-2) i_{\max }+\eta(\eta-1) \sigma-\eta \sigma}{i_{\max }+\frac{1}{2}(\eta+1) \sigma^{2}} \\
& \geq \frac{2(\eta-2) i_{\max }}{i_{\max }+\frac{1}{2}(\eta+1) \sigma^{2}}>0
\end{aligned}
$$

since $\eta>2$. Substitution (B.37) into (B.36), and using $d_{2}(\bar{x}) \geq 0$, we have

$$
\frac{\partial f_{2}\left(\bar{x}_{2}\right)}{\partial A} \frac{\partial A}{\partial \sigma}+\frac{\partial f_{2}\left(\bar{x}_{2}\right)}{\partial \eta} \frac{\partial \eta}{\partial \sigma} \geq\left(\frac{2(\eta-2) i_{\max }}{i_{\max }+\frac{1}{2}(\eta+1) \sigma^{2}}\right) A \bar{x}^{2} k_{\mathrm{s}}^{2}-\frac{\sigma \eta p\left(k_{\mathrm{b}}-k_{\mathrm{s}}\right)}{i_{\max }+\frac{1}{2}(2 \eta-1) \sigma^{2}}
$$


Finally note that $\bar{x} \geq \frac{p\left(k_{\mathrm{b}}-k_{\mathrm{s}}\right)}{B k_{\mathrm{b}}}$, so

$$
\begin{aligned}
\frac{\partial f_{2}\left(\bar{x}_{2}\right)}{\partial A} \frac{\partial A}{\partial \sigma}+\frac{\partial f_{2}\left(\bar{x}_{2}\right)}{\partial \eta} \frac{\partial \eta}{\partial \sigma} & \geq\left(\frac{2(\eta-2) i_{\max }}{i_{\max }+\frac{1}{2}(\eta+1) \sigma^{2}}\right) A\left(\frac{p\left(k_{\mathrm{b}}-k_{\mathrm{s}}\right) k_{\mathrm{s}}}{B k_{\mathrm{b}}}\right)^{2}-\frac{\sigma \eta p\left(k_{\mathrm{b}}-k_{\mathrm{s}}\right)}{i_{\max }+\frac{1}{2}(2 \eta-1) \sigma^{2}} \\
& >0
\end{aligned}
$$

by the definition of $\gamma_{4}$. Thus $\frac{\partial f_{2}\left(\bar{x}_{2}\right)}{\partial \sigma}>0$ which completes the proof.

\section{B.2 Proofs of Supporting Results}

Proof of Lemma A1. Consider an arbitrary contract $\Pi=\left(\left\{c_{t}, i_{t}\right\}, \tau\right)$ and suppose that the solution to the manager's optimization problem (A.1) for this contract is given by $\left\{\tilde{c}_{t}, \tilde{i}_{t}\right\}$ and that the manager's associated value for this contract is $\tilde{W}_{0}$.

Now, consider the alternative contract $\tilde{\Pi}=\left(\left\{\tilde{c}_{t}, \tilde{i}_{t}\right\}, \tau\right)$. Under this contract, the manager again obtains utility $\tilde{W}_{0}$ from the consumption effort pair $\left\{\tilde{c}_{t}, \tilde{a}_{t}\right\}$. We claim that the solution to the manager's optimization problem (A.1) is again $\left\{\tilde{c}_{t}, \tilde{i}_{t}\right\}$. Suppose that it is not and that there is an alternative feasible pair $\left\{\check{c}_{t}, \check{i}_{t}\right\}$ such that this policy yields the utility $\check{W}_{0}>\tilde{W}_{0}$ to the manager. The consumption effort pair $\left\{\check{c}_{t}, \check{i}_{t}\right\}$ is also feasible under the original contract $\Pi$ as

$$
\begin{aligned}
\lim _{t \rightarrow \infty} E\left[e^{-r t} \int_{0}^{t}\left(c_{s}-\check{c}_{s}\right) d s\right] & =\lim _{t \rightarrow \infty}\left(E\left[e^{-r t} \int_{0}^{t}\left(c_{t}-\tilde{c}_{t}\right) d t\right]+E\left[e^{-r t} \int_{0}^{t}\left(\tilde{c}_{s}-\check{c}_{s}\right) d s\right]\right) \\
& =\lim _{t \rightarrow \infty} E\left[e^{-r t} \int_{0}^{t}\left(c_{s}-\tilde{c}_{s}\right) d s\right]+\lim _{t \rightarrow \infty} E\left[e^{-r t} \int_{0}^{s}\left(\tilde{c}_{s}-\check{c}_{s}\right) d s\right] \\
& =0 .
\end{aligned}
$$

Thus, the manager could achieve utility $\check{W}_{t}>\tilde{W}_{t}$ under the original contract $\Pi$, a contradiction.

Finally, the investor achieves the same value under the new contract $\tilde{\Pi}$ as under the original contract $\Pi$, as effort and investment are unchanged, and the transversality condition implies that the two consumption streams have the same present value.

Proof of Lemma A2. Consider the manager's problem (A.1) and denote its optimal consumptioninvestment solution by $\left(c^{*}, i^{*}\right)$ given savings $S_{t}=\mathcal{S}$ and associated value $W_{t}\left(\Pi,\left\{X_{s}, K_{s}\right\}_{s \leq t} ; \mathcal{S}\right)$. We claim that for $\mathcal{S}=0$, a feasible plan $\left(c^{*}-r \mathcal{S}, i^{*}\right)$ solves problem (A.1). It then holds that 
$W_{t}\left(\Pi,\left\{X_{s}, K_{s}\right\}_{s \leq t} ; 0\right)=e^{\gamma r \mathcal{S}} W_{t}\left(\Pi,\left\{X_{s}, K_{s}\right\}_{s \leq t} ; \mathcal{S}\right)$. Suppose that there is some alternative $(\check{c}, \check{a})$ that yields a higher utility to the manager with zero savings. That is, $\check{W}_{t}\left(\Pi,\left\{X_{s}, K_{s}\right\}_{s \leq t} ; 0\right)>$ $W_{t}\left(\Pi,\left\{X_{s}, K_{s}\right\}_{s \leq t} ; 0\right)$. Now, consider the plan $(\check{c}+r S, \check{i})$ and note that this plan is feasible under $S_{t}=\mathcal{S}$, but that under this plan, the manager can achieve the following utility:

$$
\begin{aligned}
\check{W}_{t}\left(\Pi,\left\{X_{s}, K_{s}\right\}_{s \leq t} ; \mathcal{S}\right) & =e^{-\gamma r \mathcal{S}} \check{W}_{t}\left(\Pi,\left\{X_{s}, K_{s}\right\}_{s \leq t} ; 0\right) \\
& \geq e^{-\gamma r \mathcal{S}} W_{t}\left(\Pi,\left\{X_{s}, K_{s}\right\}_{s \leq t} ; 0\right) \\
& =W_{t}\left(\Pi,\left\{X_{s}, K_{s}\right\}_{s \leq t} ; \mathcal{S}\right) .
\end{aligned}
$$

This contradicts the optimality of $\left(c^{*}, i^{*}\right)$. Thus, $\left(c^{*}-r \mathcal{S}, i^{*}\right)$ is indeed optimal, and

$$
W_{t}\left(\Pi,\left\{X_{s}, K_{s}\right\}_{s \leq t} ; 0\right)=e^{\gamma r \mathcal{S}} W_{t}\left(\Pi,\left\{X_{s}, K_{s}\right\}_{s \leq t} ; \mathcal{S}\right)
$$

This implies the following:

$$
\frac{\partial}{\partial \mathcal{S}} W_{t}\left(\Pi,\left\{X_{s}, K_{s}\right\}_{s \leq t} ; 0\right)=-\gamma r W_{t}\left(\Pi,\left\{X_{s}, K_{s}\right\}_{s \leq t} ; 0\right)
$$

In any optimal consumption-savings plan, the manager's marginal utility of consumption must equal her utility of savings, or $u^{\prime}\left(\tilde{c}_{t}\right)=\frac{\partial}{\partial \mathcal{S}} W_{t}\left(\Pi,\left\{X_{s}, K_{s}\right\}_{s \leq t} ; 0\right)$. Substituting Equation (B.40) into this last condition and noting that $u^{\prime}\left(\tilde{c}_{t}\right)=-\gamma u\left(\tilde{c}_{t}\right)$ gives the desired result.

Proof of Lemma A3. We restrict the manager's consumption plan to satisfy the following integrability and transversality conditions:

$$
\begin{array}{r}
E\left[\int_{0}^{\infty}-e^{-r s} u\left(\tilde{c}_{s}, \tilde{i}_{s}\right) d s\right]<\infty \\
\lim _{t \rightarrow \infty} S_{t} \stackrel{\text { a.s }}{=} 0 .
\end{array}
$$

Consider an arbitrary contract $(\beta, i, \tau)$ and note that if $W_{t}$ solves Equation (A.5), then $W_{t}$ is equal to the manager's continuation utility from choosing savings $S_{t}=0$ and investment rate $i_{t}$ by construction. Suppose that $\beta_{t}$ and $i_{t}$ satisfy Equation (A.6) and consider an arbitrary policy $(\tilde{c}, \tilde{i})$. 
Let

$$
G_{t}=\int_{0}^{t} e^{-r s} u\left(\tilde{c}_{s}, \tilde{i}_{s}\right) d s+e^{-r t} e^{-\gamma r S_{t}} W_{t}
$$

where $S_{t}=\int_{0}^{t} e^{r(t-s)}\left(c_{s}-\tilde{c}_{s}\right) d s$ is the manager's accumulated savings when she chooses the alternative consumption plan. An application of Ito's lemma yields the following:

$$
e^{r t+\gamma r S_{t}} d G_{t}=\left(-\gamma r W_{t}\left(c_{t}-\tilde{c}_{t}\right)-\gamma r W_{t} \beta_{t}\left(\tilde{i}_{t}-i_{t}\right) X_{t}+e^{\gamma r S_{t}} u\left(\tilde{c}_{t}, \tilde{i}_{t}\right)\right) d t-\gamma r W_{t} \beta_{t} d Z_{t} .
$$

The $\tilde{c}_{t}$ and $\tilde{i}_{t}$ that maximize the drift term above must satisfy the following first-order conditions:

$$
\begin{aligned}
\gamma r W_{t} & =-e^{\gamma r S_{t}} u_{c}\left(\tilde{c}_{t}, \tilde{i}_{t}\right), \\
\gamma r W_{t} \beta_{t} X_{t} & =-\theta g^{\prime}(i) X_{t} K_{t} e^{\gamma r S_{t}} u_{c}\left(\tilde{c}_{t}, \tilde{i}_{t}\right),
\end{aligned}
$$

as $u_{i}\left(c_{t}, i_{t}\right)=-u_{c}\left(c_{t}, i_{t}\right) \theta g^{\prime}\left(i_{t}\right) X_{t} K_{t}$. These first-order conditions are solved for $\tilde{c}_{t}=c_{t}+r S_{t}$ and $\tilde{i}_{t}=i_{t}$, as $r W_{t}=u\left(c_{t}, i_{t}\right)$. Moreover, for $\tilde{c}_{t}=c_{t}+r S_{t}$ and $\tilde{i}_{t}=i_{t}$, the drift term is zero. Thus, for all other choices of consumption and effort, the drift term is weakly negative and $G_{t}$ is a supermartingale.

The manager's value from choosing the policy $(\tilde{c}, \tilde{i})$ is expressed as follows:

$$
\begin{aligned}
E\left[\int_{0}^{\infty} e^{-r s} u\left(\tilde{c}_{s}, \tilde{i}_{s}\right) d s\right] & =E\left[G_{t}\right]+E\left[\int_{t}^{\infty} e^{-r s} u\left(\tilde{c}_{s}, \tilde{i}_{s}\right) d s-e^{-r t-\gamma r S_{t}} W_{t}\right] \\
& \leq G_{0}+E\left[\int_{t}^{\infty} e^{-r s}\left(u\left(\tilde{c}_{s}, \tilde{i}_{s}\right)-e^{\gamma r S_{t}} u\left(c_{s}, i_{s}\right)\right) d s\right]
\end{aligned}
$$

Now note that $\lim _{t \rightarrow \infty} S_{t} \stackrel{\text { a.s. }}{=} 0$ such that $\lim _{t \rightarrow \infty}\left|\tilde{c}_{t}-c_{t}\right| \stackrel{a . s .}{=} 0$, which in turn implies the following:

$$
\lim _{t \rightarrow \infty} \int_{t}^{\infty} e^{-r s}\left(u\left(\tilde{c}_{s}, \tilde{i}_{s}\right)-e^{\gamma r S_{t}} u\left(c_{s}, i_{s}\right)\right) d s \stackrel{a . s}{=} 0
$$

Finally, by the condition given in Equation (B.41) and Fubini's theorem, we can take the limit as $t \rightarrow \infty$ of both sides of Equation (B.44) to obtain the following:

$$
\begin{aligned}
E\left[\int_{0}^{\infty} e^{-r s} u\left(\tilde{c}_{s}, \tilde{i}_{s}\right) d s\right] & \leq G_{0}+\lim _{t \rightarrow \infty} E\left[\int_{t}^{\infty} e^{-r s}\left(u\left(\tilde{c}_{s}, \tilde{i}_{s}\right)-e^{\gamma r S_{t}} u\left(c_{s}, i_{s}\right)\right) d s\right] \\
& =G_{0}=W_{0} .
\end{aligned}
$$


Thus, all other consumption and effort plans $\left(\tilde{c}_{t}, \tilde{i}_{t}\right)$ yield no more utility than $\left(c_{t}, i_{t}\right)$, and the contract is incentive-compatible and zero-savings.

The conditions given are necessary for a contract to be zero-savings according to Lemma A2. To see that the conditions are also necessary for incentive compatibility, consider any contract such that $\beta_{t}$ does not satisfy the condition given in Equation (A.6). The same argument given above would show that the optimal response to such a contract would be to choose $\tilde{i}_{t} \neq i_{t}$.

Proof of Lemma A4. Let

$$
L=\left(\frac{\eta-1}{\eta}\right)\left(\frac{k_{\mathrm{b}}}{k_{\mathrm{s}}}\right)^{2}\left(\frac{k_{\mathrm{b}}}{r}-B k_{\mathrm{s}}\right)\left(\frac{\gamma x_{\mathrm{b}}^{*}}{k_{\mathrm{b}}-k_{\mathrm{s}}}\right)
$$

Note that $L$ does not depend on $\gamma$ because the dependence on $\gamma$ in the term $\gamma x_{\mathrm{b}}^{*}$ cancels out.

Let $\hat{V}$ solve

$$
r \hat{V}=x k_{\mathrm{s}}\left(1-\theta i_{\max }\right)+i_{\max } x \hat{V}_{x}+\frac{1}{2} x^{2} \hat{V}_{x x}
$$

such that

$$
\begin{aligned}
\hat{V}(0) & =0, \\
\hat{V}(\hat{X}) & =V\left(x, k_{\mathrm{b}}\right)-p\left(k_{\mathrm{b}}-k_{\mathrm{s}}\right), \\
\hat{V}_{x}(\hat{X}) & =V_{x}\left(x, k_{\mathrm{b}}\right) .
\end{aligned}
$$

Observe that $\hat{x}$ is finite, as $k_{\mathrm{b}}\left(r-i_{\max }\right)>k_{\mathrm{s}} r\left(1-\theta i_{\max }\right)$. Suppose that $\hat{x} \geq x_{\mathrm{b}}^{*}$; then, we can combine Equations (B.48) and (B.49) to obtain the following:

$$
\left(\frac{k_{\mathrm{b}}}{r}-B k_{\mathrm{s}}\right) \hat{x}+\left(\frac{\eta+\varepsilon}{\eta-1}\right) \mathcal{C}_{\mathrm{b} 2} \hat{x}^{-\varepsilon}=p\left(\frac{\eta}{\eta-1}\right)\left(k_{\mathrm{b}}-k_{\mathrm{s}}\right)
$$


As $\eta>1, \varepsilon>0$, and $\mathcal{C}_{\mathrm{b} 2}>0$, Equation (B.50) implies the following:

$$
\begin{aligned}
\hat{x} & \leq p\left(\frac{\eta}{\eta-1}\right)\left(k_{\mathrm{b}}-k_{\mathrm{s}}\right)\left(\frac{k_{\mathrm{b}}}{r}-B k_{\mathrm{s}}\right)^{-1} \\
& \leq\left(\frac{L}{\gamma}\right)\left(\frac{\eta}{\eta-1}\right)\left(k_{\mathrm{b}}-k_{\mathrm{s}}\right)\left(\frac{k_{\mathrm{b}}}{r}-B k_{\mathrm{s}}\right)^{-1} \\
& =\left(\frac{k_{\mathrm{b}}}{k_{\mathrm{s}}}\right)^{2} x_{\mathrm{b}}^{*} .
\end{aligned}
$$

As $\hat{V}(x) \geq V\left(x, k_{\mathrm{s}}\right), \bar{x} \leq \hat{x} \leq\left(\frac{k_{\mathrm{b}}}{k_{\mathrm{s}}}\right)^{2} x_{\mathrm{b}}^{*}$. Furthermore, $i_{\max } x_{\mathrm{b}}^{*} V\left(x_{\mathrm{b}}^{*}, k_{\mathrm{b}}\right)=\theta i_{\max } x_{\mathrm{b}}^{*} k_{\mathrm{b}}+$ $\frac{1}{2} \gamma r\left(\theta \sigma x_{\mathrm{b}}^{*} k_{\mathrm{b}}\right)^{2}$ and $V\left(x, k_{\mathrm{b}}\right)$ is convex for $x>x_{\mathrm{b}}^{*}$. Thus, the smooth-pasting condition for $\bar{x}$ in Equation (A.13) implies the following:

$$
\begin{aligned}
i_{\max } \bar{x} V_{x}\left(\bar{x}, k_{\mathrm{s}}\right) & =i_{\max } \bar{x} V_{x}\left(\bar{x}, k_{\mathrm{b}}\right) \\
& \geq i_{\max } \bar{x} V_{x}\left(x_{\mathrm{b}}^{*}, k_{\mathrm{b}}\right) \\
& =\frac{\bar{x}}{x_{\mathrm{b}}^{*}}\left(i_{\max } \theta x_{\mathrm{b}}^{*} k_{\mathrm{b}}+\frac{1}{2} \gamma r\left(\theta \sigma x_{\mathrm{b}}^{*} k_{\mathrm{b}}\right)^{2}\right) \\
& =i_{\max } \theta \bar{x} k_{\mathrm{b}}+\frac{1}{2} \bar{x} x_{\mathrm{b}}^{*} \gamma r\left(\theta \sigma k_{\mathrm{b}}\right)^{2} \\
& \geq i_{\max } \theta \bar{x} k_{\mathrm{s}}+\frac{1}{2} \gamma r\left(\theta \sigma \bar{x} k_{\mathrm{s}}\right)^{2},
\end{aligned}
$$

which implies that $i^{*}\left(\bar{x}, k_{\mathrm{s}}\right)=i_{\max }$, the desired result.

Proof of Lemma A5. First, note that it is straightforward to see from Equation (A.33) that $\bar{x}_{2}$ is increasing in $\gamma$ and from Equation (A.21) that $x_{\mathrm{b}}^{*}$ is decreasing in $\gamma$. Moreover, $\left.\bar{x}_{1}\right|_{\gamma=0}=\bar{x}^{F B}<$ $\lim _{\gamma \rightarrow 0} x_{\mathrm{b}}^{*}=\infty$ and $\lim _{\gamma \rightarrow \infty} x_{\mathrm{b}}^{*}=0<\lim _{\gamma \rightarrow 0} \bar{x}_{1}=\infty$. Thus, there exists a unique $\gamma_{1}$ such that $\bar{x}_{1} \leq x_{\mathrm{b}}^{*}$ if and only if $\gamma \leq \gamma_{1}$ and $\left.\bar{x}_{1}\right|_{\gamma=\gamma_{1}}=\left.x_{\mathrm{b}}^{*}\right|_{\gamma=\gamma_{1}}$. Note that $\left.\bar{x}_{1}\right|_{\gamma=\gamma_{1}}=\left.x_{\mathrm{b}}^{*}\right|_{\gamma=\gamma_{1}}$ also implies that $d_{1}\left(\left.\bar{x}_{1}\right|_{\gamma=\gamma_{1}}\right)=d_{2}\left(\left.\bar{x}_{1}\right|_{\gamma=\gamma_{1}}\right)=1$, so that Equations (A.30) and (A.31) are equivalent and $\left.\bar{x}_{1}\right|_{\gamma=\gamma_{1}}=\left.\bar{x}_{2}\right|_{\gamma=\gamma_{1}}$. If $\gamma>\gamma_{1}$, then the only possible solution is $\bar{x}_{2}$. 


\section{Appendix C: Additional Results}

\section{C.1 The Complementarity of Incremental and Lumpy Capital}

Our goal here is to clarify that the results of the paper are due to the dynamics of the problem rather than simply due to a technological assumption of substitutability between inputs in our production and profit functions. To this end, we show that incremental and lumpy capital are production complements in the static version of the investment problem faced by investors. Consider the following problem

$$
\max _{i \geq 0, K \in\left\{k_{s}, k_{b}\right\}}\left\{X(1+i) K-\theta g(i) X K-P \mathbb{1}\left(K=k_{b}\right)\right\}
$$

where $i$ is investment in $X$ and $g(i)$ an investment cost function for $X$ with $g^{\prime}(i) \geq 0$ and $g^{\prime \prime}(i) \geq 0$ for $i \geq 0$. This problem assumes that the firm is endowed with $k_{s}$ units of capital and can add $k_{b}-k_{s}$ units of capital at total cost $P$. We want to determine how the value of the additional $k_{b}-k_{s}$ units of capital, denoted by $\Delta_{K} V$, depends on the cost of investment in $X$ given by $\theta$. It is optimal to invest in additional capital if and only if $\Delta_{K} V \geq P$. Thus, if $\Delta_{K} V$ is decreasing in $\theta$, then $X$ and $K$ are complements in that raising the cost of investment in $X$ decreases investment in $K$.

First, we take a first-order condition of Problem (C.1) with respect to $i$ to determine the optimal investment in $X$

$$
g^{\prime}\left(i^{*}\right)=\frac{1}{\theta}
$$

Note that optimal investment in $X, i^{*}$, does not depend on $K$. Moreover, this first-order condition implies that $i^{*}$ is decreasing in $\theta$ because $g^{\prime \prime}(i) \geq 0$. Next, we take the difference of the production function $X\left(1+i^{*}\right) K-\theta g\left(i^{*}\right) X K$ evaluated at $k_{b}$ and $k_{s}$ to obtain

$$
\Delta_{K} V=\left(X\left(1+i^{*}\right)-\theta g\left(i^{*}\right) X\right)\left(k_{b}-k_{s}\right)
$$

Finally, we take a derivative of $\Delta_{K} V$ with respect to $\theta$ and apply the first-order condition for $i^{*}$ to 
obtain

$$
\frac{d}{d \theta} \Delta_{K} V=\left(\frac{\partial i^{*}}{\partial \theta} X-g\left(i^{*}\right) X-\theta g^{\prime}\left(i^{*}\right) \frac{\partial i^{*}}{\partial \theta} X\right)\left(k_{b}-k_{s}\right)=-g\left(i^{*}\right) X\left(k_{b}-k_{s}\right) \leq 0,
$$

so that $\Delta_{K} V$ is decreasing in $\theta$. This implies that increasing $\theta$ decreases optimal investment in both $X$ and $K$ for all $\theta$, and thus the two inputs are complements. The intuition is that with larger $\theta$, the firm will optimally operate at a lower productivity $X$, which makes capital less productive and hence reduces investment in capital.

Note that we reach the same conclusion by considering the cross effect of inputs on the value function. The marginal effect of $X$ on the incremental benefit of investment in $K$, given by

$$
\frac{d}{d X} \Delta_{K} V=\left(1+i^{*}-\theta g\left(i^{*}\right)\right)\left(k_{b}-k_{s}\right)
$$

is always positive at the optimal $i^{*}$ assuming that the value function is weakly positive. Thus, the incremental benefit of investment in $K$ increases in the amount of $X$, in other words, the value function is supermodular in $X$ and $K$, which indicates that the two inputs are production complements.

\section{C.2 Partially Observable Shocks to Incremental Capital}

We assume that shocks to incremental capital $Z_{t}$ are given by the following:

$$
d Z_{t}=\sqrt{\alpha} d Z_{t}^{1}+\sqrt{1-\alpha} d Z_{t}^{2}
$$

where $Z_{t}^{1}$ and $Z_{t}^{2}$ are independent Brownian motions. Importantly, the investor observes $Z_{t}^{2}$ but not $Z_{t}^{1}$. Thus, $\alpha$ measures the severity of the moral hazard problem that the manager faces. When $\alpha=0$, the manager's actions are perfectly observable and there is no moral hazard problem. When $\alpha=1$, the manager's actions are as unobservable as possible and the moral hazard problem is severe. We can think of $Z_{t}^{2}$ as an industry- or market-wide shock to productivity, whereas $Z_{t}^{1}$ is a shock idiosyncratic to the firm.

The solution to the optimal contracting problem with partially observable shocks is similar to the one of the baseline moral hazard model in Appendix A.1. In the following, we highlight the 
necessary adoptions.

Lemmas A1 and A2 hold in the current setup. To derive the dynamics of $W_{t}$ in an incentivecompatible zero-savings contract with partially observable shocks, the martingale representation theorem implies that there exist two progressively measurable processes $\beta_{t}$ and $\lambda_{t}$ such that the following holds:

$$
d M_{t}=-\gamma r W_{t} e^{-r t}\left(\beta_{t}\left(d X_{t}-i_{t} X_{t} d t-\sigma \sqrt{1-\alpha} X_{t} d Z_{t}^{2}\right)+\lambda_{t} \sigma \sqrt{1-\alpha} X_{t} d Z_{t}^{2}\right)
$$

This yields the following dynamics for the manager's continuation utility under the recommended consumption and investment plan:

$$
d W_{t}=-\gamma r W_{t}\left(\beta_{t}\left(d X_{t}-i_{t} X_{t} d t-\sigma \sqrt{1-\alpha} X_{t} d Z_{t}^{2}\right)+\lambda_{t} \sigma \sqrt{1-\alpha} X_{t} d Z_{t}^{2}\right) .
$$

Because a deviation from the recommended $i$ offers the same costs and benefits as those in Appendix A.1, it can be readily verified that the incentive-compatible, zero-savings $\beta_{t}$ must satisfy condition (A.7), whereas $\lambda_{t}$ is any progressively measurable process.

It is again convenient to use the certainty equivalent of the manager's continuation utility as a state variable for the investor's problem. Applying Ito's lemma to $Y_{t}=-1 /(\gamma r) \ln \left(-\gamma r W_{t}\right)$ and Equation (C.2) yields that the dynamics of $Y_{t}$ under an incentive-compatible zero-savings contract are given by the following:

$$
d Y_{t}=\frac{1}{2} \gamma r \sigma^{2}\left(\alpha \beta_{t}^{2}+(1-\alpha) \lambda_{t}^{2}\right) X_{t}^{2} d t+\sigma X_{t}\left(\sqrt{\alpha} \beta_{t} d Z_{t}^{1}+\sqrt{1-\alpha} \lambda_{t} d Z_{t}^{2}\right)
$$

where $\beta_{t}$ is given by Equation (A.7).

The remaining steps of Appendix A.1 are easily adapted. As before, the investor would never expose the manager to more risk than is required to provide incentives. Thus, the sensitivity to observable shocks $\lambda_{t}$ is always zero. It follows that the total firm value function under the optional contract must satisfy the HJB equation, Equation (19), where the incentive cost is given by the following:

$$
\rho(i, x, k)=\frac{1}{2} \mathbb{1}(i>0) \alpha \gamma r\left(\theta \sigma g^{\prime}(i) x k\right)^{2},
$$


which differs from the incentive cost in the baseline moral hazard model in Equation (20) by the linear dependence on $\alpha$. This shows that the observability parameter $\alpha$ affects the contract in exactly the same way as the coefficient of the manager's risk aversion $\gamma$. A straightforward adaptation of Proposition 4 verifies the optimality of the proposed contract under the partial observability of shocks.

\section{C.3 Single-capital Version of the Model}

In this appendix, we analyze the version of the model of Section 2 with only one form of capital and abstract away from incremental capital. Consider a firm that can invest in capital $K$ continuously at rate $I$ subject to adjustment $\operatorname{cost} F(I, k)$ and discretely with a one-time increase by fraction $\Delta$ subject to a fixed cost $P$. In addition, capital $K$ depreciates at rate $\delta$. Thus, the capital stock evolves as follows:

$$
d K_{t}=\left(I_{t}-\delta_{t} K_{t}\right) d t+\sigma K_{t} d Z_{t}+\Delta K_{t} \mathbb{1}(t=\tau)
$$

where $\sigma$ is the volatility of the capital accumulation shock. Using $i_{t}=I_{t} / K_{t}, d K_{t}$ can be written as follows:

$$
d K_{t}=\left(i_{t}-\delta\right) K_{t} d t+\sigma K_{t} d Z_{t}+\Delta K_{t} \mathbb{1}(t=\tau)
$$

We assume that the choice of $i$ is constrained to $\left[0, i_{\max }\right]$ for some positive $i_{\max }<r$. One unit of capital generates one dollar of cash flow. The firm's cash flows net of the investment costs are then given by the following:

$$
K_{t} d t-\theta G\left(i_{t}, K_{t}\right) d t-P \mathbb{1}(t=\tau)
$$

where $G(i, k)=F(I, k)$. The problem is very similar in its structure to that of Section 2 . Under some regularity condition, the optimal policy for lumpy investment takes the form of an upper threshold $\bar{k}$. Denote the time to invest by $\tau=\inf \left\{t: K_{t} \geq \bar{k}\right\}$.

For simplicity, assume that $\delta=0$. Standard arguments suggest that post-lumpy-investment firm value, denoted by $V_{\mathrm{b}}(k)$, must satisfy the following HJB equation:

$$
r V_{\mathrm{b}}=\max _{i} k-\theta G(i, k)+i k V_{\mathrm{b}}^{\prime}+\frac{1}{2} \sigma^{2} k^{2} V_{\mathrm{b}}^{\prime \prime}
$$


Pre-lumpy-investment firm value, denoted by $V_{\mathrm{S}}(k)$, solves a similar HJB equation:

$$
r V_{\mathrm{s}}=\max _{i} k-\theta G(i, k)+i k V_{\mathrm{s}}^{\prime}+\frac{1}{2} \sigma^{2} k^{2} V_{\mathrm{s}}^{\prime \prime}
$$

subject to boundary conditions at $k=\bar{k}$ :

$$
\begin{gathered}
V_{\mathrm{s}}(\bar{k})=V_{\mathrm{b}}((1+\Delta) \bar{k})-P, \\
V_{\mathrm{s}}^{\prime}(\bar{k})=(1+\Delta) V_{\mathrm{b}}^{\prime}((1+\Delta) \bar{k}) .
\end{gathered}
$$

If interior, the optimal investment rate $i_{j}^{*}$ solves the following:

$$
\theta G_{i}\left(i_{j}^{*}, k\right)=k V_{j}^{\prime}(k)
$$

where the subscript $j \in\{\mathrm{s}, \mathrm{b}\}$ denotes the pre- and post-lumpy-investment values.

It is common to assume that $F(I, k)$ is homogeneous of degree one in $I$ and $k$, which means that $G(i, k)$ is separable in $i$ and $k, G(i, k)=g(i) k$. As in the model of Section 2, the cost function $g(i)$ can encompass the direct and adjustment costs of investment. If $g(i)=i$, it captures only the direct cost of investment in capital. An increasing and convex $g(i)$ captures the adjustment costs.

Problem (C.3)-(C.6) is isomorphic to the one studied in Sections 2 and 3. Thus, we can readily obtain results analogous to Propositions 2 and 3. The model can also be extended to incorporate moral hazard analogous to Section 4.

\section{C.4 Option to Abandon}

In this section, we consider a firm that holds an option to abandon its operations. The setup is identical to that in Sections 2.1 and 4.1, except that instead of holding an option to increase capital, the firm starts with lumpy capital $k_{0}$ and can now liquidate and sell all of its capital for a fixed price $Q$. We assume that the firm honors the promised obligations to the manager after liquidation. The problems of investment in incremental capital and of providing the manager with incentives are essentially identical to those one analyzed in the main text for the case of a growth option. The total value of the firm before liquidation is denoted by $V(x)$. Before liquidation, the 
value of the firm and optimal contract are given by the solution to the following HJB equation:

$$
r V=\max _{i \in\left[0, i_{\max }\right]}\left\{x k_{0}(1-\theta g(i))+i x V^{\prime}+\frac{1}{2} \sigma^{2} x^{2} V^{\prime \prime}-\rho(i, x)\right\}
$$

where

$$
\rho(i, x)=\frac{1}{2} \mathbb{1}(i>0) \gamma r\left(\theta \sigma g^{\prime}(i) x k_{0}\right)^{2}
$$

The equation is essentially the same as Equation (19), but it requires a set of different boundary conditions that are consistent with the option to abandon. As expected, the optimal exercise policy takes the form of a lower threshold $\underline{x}$ such that the firm liquidates the first time that $X_{t}$ is at or below $\underline{x}$. At $x=\underline{x}$, the following value-matching and smooth-pasting conditions must hold:

$$
\begin{aligned}
V(\underline{x}) & =Q \\
V^{\prime}(\underline{x}) & =0 .
\end{aligned}
$$

As $x$ approaches infinity, the probability of abandonment approaches zero. As the incentive cost of effort $\rho$ (quadratic in $x$ ) increases more rapidly than cash flows (linear in $x$ ), $i$ approaches zero. Thus, $V(x)$ becomes a linear function consistent with $i=0$ as $x$ approaches infinity:

$$
\lim _{x \rightarrow \infty} V^{\prime}(x)=\frac{1}{r}
$$

The optimality of the solution to Equations (C.8)-(C.12) can be verified by a straight-forward adaptation of the proof of Proposition 4.

The main takeaway of this model is that an increase in the severity of the moral hazard problem increases the optimal liquidation threshold $\underline{x}$. This result is intuitive, as an increase in moral hazard decreases the value of the firm before liquidation but does not affect the liquidation value $Q$ and thus makes early liquidation more attractive. This intuition mirrors the intuition that we present in the main model with a growth option in that the effect of moral hazard on the timing of the option exercise depends on the differences in sensitivity to the moral hazard of the values before and after the option exercise. In the case of an option to abandon, this mechanism leads to the unambiguous acceleration of liquidation with the increasing severity of moral hazard. We interpret 
this type of early liquidation as a form of underinvestment due to moral hazard. 